\title{
CURVAS DE REFERÊNCIA NÃO LINEARIZÁVEIS BASEADAS EM DADOS LONGITUDINAIS
}

\section{Alexandre Ryuzo Shinzato}

Dissertação apresentada ao Instituto de Matemática e Estatística da Universidade de São Paulo para obtenção do grau de Mestre em Estatística

Area de concentraçao: Estatística Orientador: Prof. Dr. Julio da Motta Singer

- São Paulo - 2004 - 


\section{CURVAS DE REFERÊNCIA NÃO-LINEARIZÁVEIS BASEADAS EM DADOS LONGITUDINAIS}

\section{Este exemplar corresponde à redação final da dissertação devidamente corrigida e defendida por Alexandre Ryuzo Shinzato e aprovada pela comissão julgadora.}

São Paulo, dezembro de 2004.

Banca Examinadora:

- Prof. Dr. Júlio da Motta Singer (Orientador) - IME-USP

- Prof. Dr. Antonio Carlos Pedroso de Lima - IME-USP

- Prof. Dra. Solange Andreoni - UNIFESP 
A meus pais Zelia e Nelson, a meu irmão Marcos,

a minha namorada Selma e amigos. 


\section{Agradecimentos}

Agradeço primeiramente a Deus por sua presença sentida ao longo de minha vida. Agradeço a todos os fatos que contribuíram para a minha formação como pessoa e estatístico.

Agradeço aos meus pais Zelia e Nelson e ao meu irmão Marcos, pela dedicação, pelo amor e o apoio incondicional em todos os momentos da minha vida.

Aos meus mestres, em particular os professores Julio da Motta Singer e Clovis de Araújo Peres cuja paciência, orientação e dedicação permitiram o meu crescimento profissional e pessoal. A todos, minha eterna gratidão, respeito, apoio e consideração.

Agradeço a minha namorada Selma, cujo apoio, orientação e paciência contribuiu de forma significativa para o bom término de mais essa etapa em minha vida.

Aos meus antigos amigos da Escola Paulista de Medicina e aos meus novos amigos da Telefônica que dividiram os momentos de aflição e alegria.

Aos meus amigos do IME, que entenderam a minha ausência dos domingos de cerveja e futebol. Enfim a todos amigos que contribuíram de alguma forma para a concretização deste trabalho. 


\title{
Resumo
}

Intervalos de referência são usados para detectar a ocorrência de valores extremos e/ou inesperados na mensuração de certas características biométricas e sua especificação é feita pela estimação de percentis. Este trabalho considera a construção de intervalos de referência baseados em dados longitudinais em situações nas quais a relação entre a característica de interesse e a variável explicativa é não linear. Nestas situações, os intervalos de referência são determinados pela estimação de curvas de referência que podem ser calculadas como funções dos parâmetros de um modelo não linear hierárquico.

\begin{abstract}
Reference intervals are used to detect the occurrence of extreme and/or unexpected values in measuring certain biometric characteristics and its specification is done by the estimation of centiles. This research considers construction of reference intervals based in longitudinal data in situations where the relation between interest characteristic and explanatory variable is nonlinear. In such situations, reference intervals are specified by reference curves that may be obtained through a nonlinear hierarchical model.
\end{abstract}




\section{Sumário}

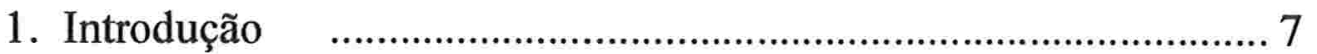

2. Modelos Não Linearizáveis para Dados Longitudinais $\quad$.............. 13

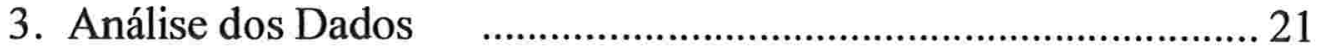

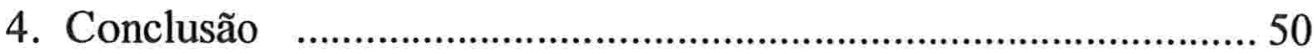




\section{Capítulo 1}

\section{Introdução}

Intervalos de referência são usados para detectar a ocorrência de valores extremos e/ou inesperados na mensuração de certas características biométricas (altura, peso, concentração de colesterol, triglicérides, etc.). Esses valores podem estar associados a pessoas com maior risco de disfunções e/ou doenças e têm sido utilizados como ferramenta auxiliar para diagnóstico e tratamento (Altman, 1990).

Um intervalo de referência de $100 \gamma \%(0<\gamma<1)$ para uma certa característica consiste de uma faixa de valores em torno de sua mediana abrangendo $100 \gamma \%$ dos dados. Por exemplo, um intervalo de referência de $95 \%$ contém valores compreendidos entre os percentis $2,5 \%$ e $97,5 \%$ da distribuição da característica de interesse (Royston and Matthews, 1991). Em geral, a construção desses intervalos envolve técnicas estatísticas bastante difundidas na literatura pertinente (Gräsbeck and Saris, 1969; Harris and DeMets, 1972).

Em alguns casos, no entanto, a construção dos intervalos de confiança é mais complexa, pois envolve outras características além daquela de interesse. Quando essa outra característica corresponde a uma variável categorizada, pode-se construir intervalos de referência para cada categoria da variável. No caso de a outra característica envolvida ser uma variável contínua, pode-se construir intervalos de referência condicionados aos valores observados. Se por exemplo, a característica de interesse for relacionada com a idade, esses intervalos são denominados intervalos de 
referência específicos à idade (age specific reference intervals - Royston, 1991).

Frequentemente, os intervalos de referência são construídos a partir de dados obtidos da população de interesse por intermédio de amostras transversais. Nessas pesquisas, a composição da amostra é baseada numa única observação de cada indivíduo para cada característica biométrica avaliada. Wright and Royston (1999) apresentam um panorama das diferentes metodologias utilizadas para a construção de intervalos de referência.

Esses intervalos são adequados para a identificação de valores não usuais da característica biométrica de interesse, mas não servem para avaliar sua evolução para um determinado indivíduo (Fujita, 1998). Em estudos longitudinais, várias observações da característica biométrica de interesse são obtidas no mesmo indivíduo e o conjunto dessas observações estabelece um padrão ou perfil individual que corresponde à evolução dessa característica de interesse.

A variabilidade presente nos dados oriundos de estudos transversais tem como fonte apenas as diferenças entre indivíduos. A variabilidade presente em dados longitudinais é proveniente de duas fontes: (i) diferenças entre indivíduos (variabilidade entre indivíduos), e (ii) diferenças entre observações realizadas no mesmo indivíduo (variabilidade intra-indivíduos) (Diggle et. al, 2002).

Quando o entendimento da variabilidade intra-indivíduos é importante, como ocorre em muitos estudos na área de medicina, intervalos de referência devem ser construídos a partir de dados oriundos de estudos longitudinais. Intervalos de referência assim construídos serão aqui denominados curvas de referência (Sañudo, 2000). Essas curvas são tomadas como referência para a avaliação da evolução da distribuição da variável de interesse como função de outra variável explicativa como a 
idade ou o tempo e são ferramentas que têm finalidade análoga a dos intervalos de referência específicos à idade. Curvas de referência servem para detectar a ocorrência de valores ou perfis individuais extremos e/ou inesperados na mensuração da característica biométrica de interesse.

Intervalos específicos à idade são construídos a partir de estudos transversais e não levam em consideração nem a correlação existente entre observações tomadas no mesmo indivíduo, nem a variabilidade das mesmas (variabilidade intra-indivíduos); as curvas de referência, por outro lado levam esses aspectos em consideração.

Royston (1995) apresenta uma metodologia para a construção das curvas de referência em situações em que a relação entre a variável de interesse e a preditora é linear ou linearizável. Ele também apresenta um método baseado em simulação para a construção dos respectivos intervalos de confiança. Singer e Shinzato (1999) constroem curvas de referência para dados com estrutura de covariância distinta daquela apresentada por Royston (1995); Sañudo (2000) estuda um método analítico para a obtenção dos intervalos de confiança para as curvas de referência. Nesses casos, modelos lineares com efeitos aleatórios são utilizados.

Existem situações mais gerais em que a metodologia citada acima não é adequada. Essas situações envolvem relações não linearizáveis [intrinsecamente não lineares, na denominação de Ratkowsky (1989)] entre a variável de interesse e a preditora. A seguir descreve-se um estudo realizado na Escola de Educação Física e Esporte da Universidade de São Paulo (EEFE - USP) com essas características.

Vinte e dois atletas foram orientados a correr em ritmo constante pré-determinado por 6 minutos. Do inicio da corrida até o sexto minuto, o consumo de oxigênio por unidade de tempo $\left(\mathrm{VO}_{2}\right)$ foi observado. $\mathrm{O}$ conjunto das medidas tomadas no mesmo indivíduo estabelece um perfil 
individual que corresponde a uma curva do $\mathrm{VO}_{2}$ em função do tempo. $\mathrm{Na}$ Figura 1.1, curvas típicas do $\mathrm{VO}_{2}$ obtidas nesse estudo são apresentadas.

$\mathrm{Na}$ literatura pertinente [Ocel (1997), por exemplo], os perfis individuais de $\mathrm{VO}_{2}$ em experimentos similares, são descritos por um processo de três fases, que podem ser visualizados na Figura 1.2. As fases são:

- Fase I - compreende o período em que há um rápido e breve aumento no $\mathrm{VO}_{2}$, reflexo do aumento da freqüência cardíaca no organismo;

- Fase II - compreende o período em que o $\mathrm{VO}_{2}$ aumenta devido ao aumento na taxa de utilização de oxigênio nas células;

- Fase III - compreende o período em que o $\mathrm{VO}_{2}$ entra em equilíbrio com a demanda gerada pela atividade física.

Nesses estudos, a Fase I não é analisada. Essa fase é considerada como adaptação e não fornece subsídios para o entendimento dos ganhos decorrentes da prática de atividade física regular. É difícil reconhecer quando a Fase I termina e a Fase II começa. Recomenda-se desprezar os dados correspondentes aos 20 segundos iniciais da atividade física (Fukuba et al., 2002).

Durante a Fase II, a análise da variação entre o $\mathrm{VO}_{2}$ num determinado instante e o $\mathrm{VO}_{2}$ basal $\left(\Delta \mathrm{VO}_{2}\right)$ fornece subsídios para o entendimento dos ganhos decorrentes da prática de atividade física regular. Segundo Fukuba et al. (2002), o valor do $\mathrm{VO}_{2}$ no tempo $t$, após 20 segundos iniciais da atividade física pode ser expresso por

$$
V O_{2}(t)=V O_{2}(0)+\beta \cdot\left[1-\exp \left(-\frac{t-\delta}{\tau}\right)\right]
$$

em que $\beta$ é interpretado como o valor de $\Delta \mathrm{VO}_{2}$ ao atingir-se a Fase III e a soma dos parâmetros $\delta$ e $\tau$ corresponde ao tempo em que o valor de $\Delta \mathrm{VO}_{2}$ atinge $63 \%$ de $\beta$. 
Figura 1.1. Perfis individuais de consumo de oxigênio para os atletas 6,14 e 18 .

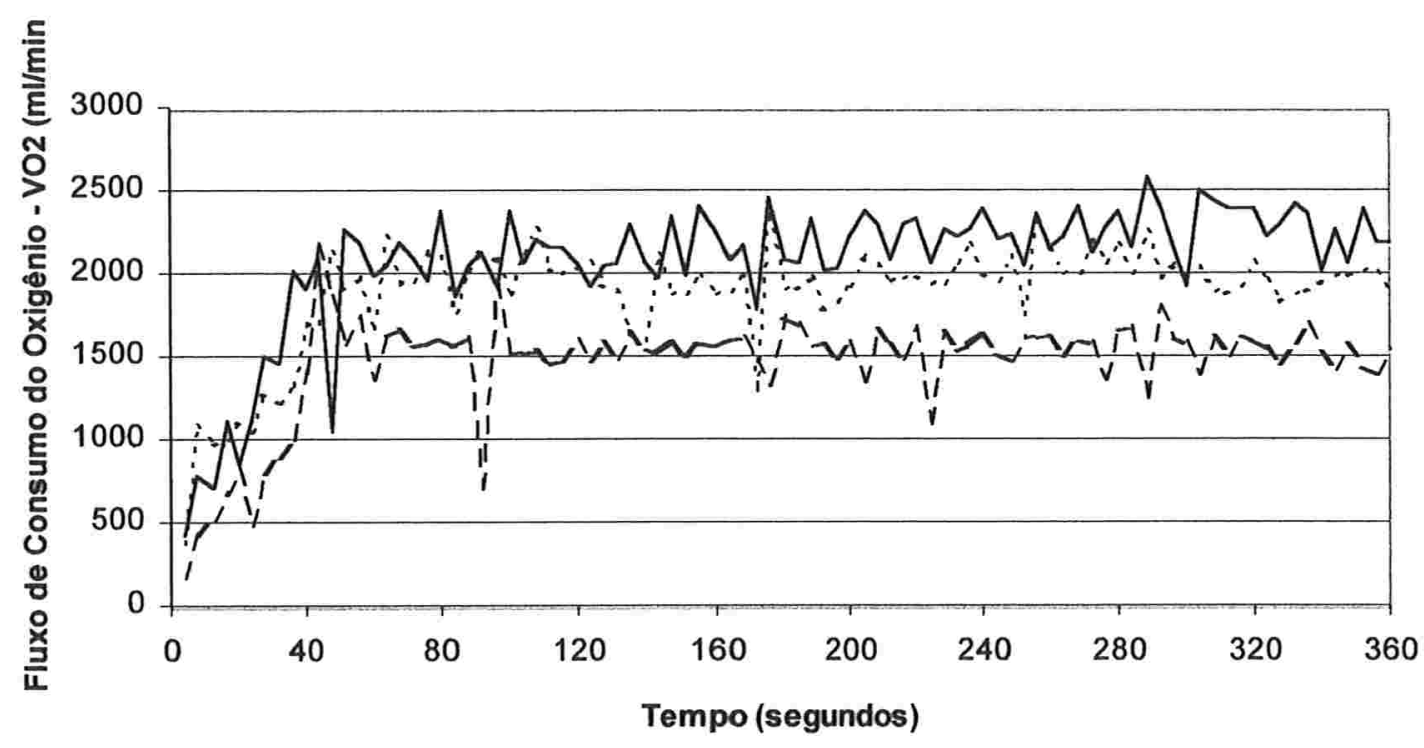
atleta 6 atleta 14 _. - atleta 18

Figura 1.2. Consumo de oxigênio durante a transição entre o repouso e o estado de equilíbrio.

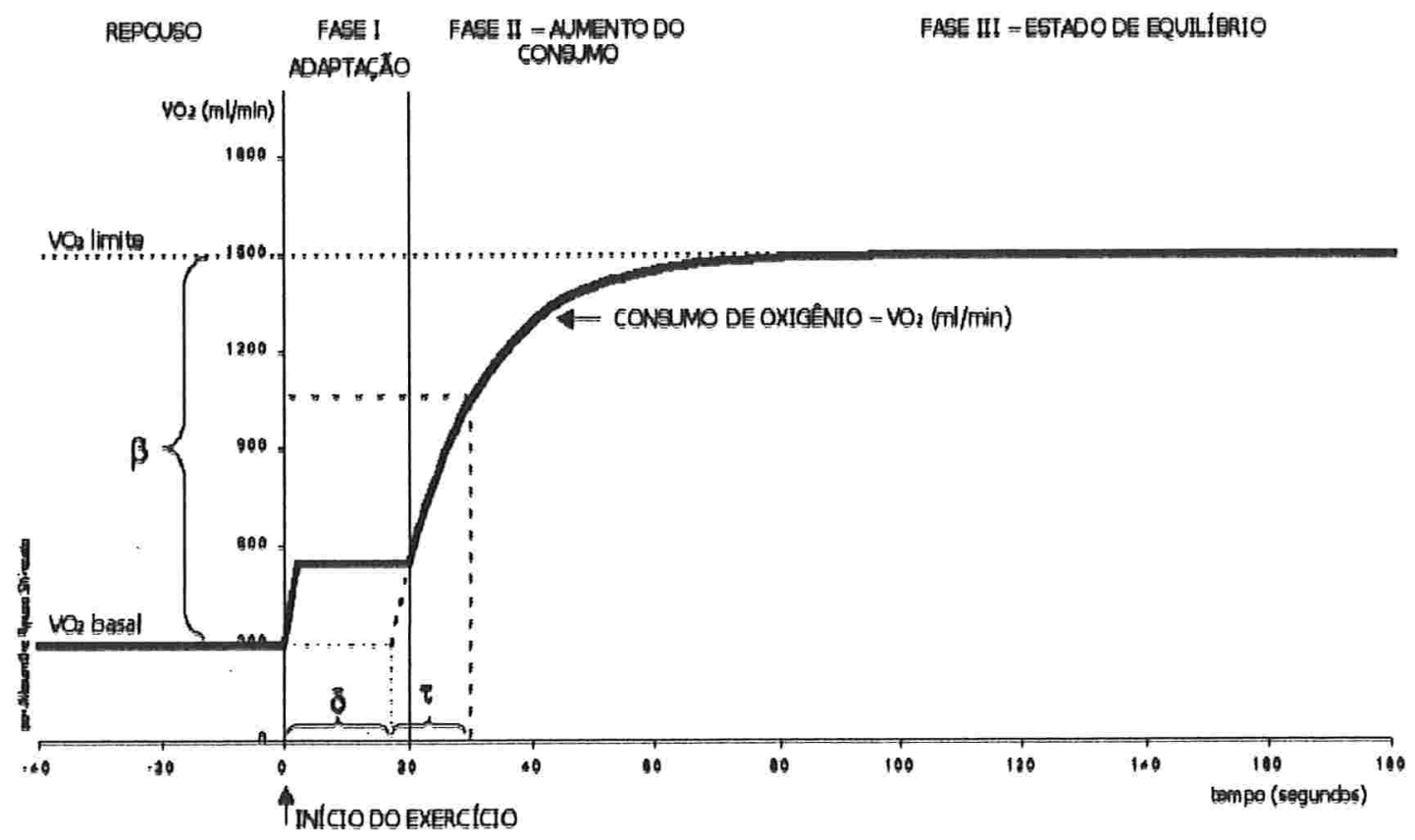


Por questões de conveniência, utilizaremos um modelo para $\Delta V O_{2}(t)=V O_{2}(t)-V O_{2}(0)$ em que o tempo é contado após 20 segundos iniciais da atividade física, que pode ser expresso por

$$
\Delta V O_{2}(t)=\beta \cdot\left[1-\exp \left(-\frac{t-\delta}{\tau}\right)\right] .
$$

Fukuba et al. (2002) substitui o $\mathrm{VO}_{2}(0)$ por uma estimativa para o $\mathrm{VO}_{2}$ basal, o consumo médio do indivíduo em estado de repouso. Neste trabalho, utilizaremos uma estimativa grosseira para o $\mathrm{VO}_{2}$ basal, a média das três primeiras observações de $\mathrm{VO}_{2}$. Este estimador pode apresentar um viés, pois utilizamos observações do consumo de oxigênio obtidas durante a fase de adaptação, porém consideramos que isso não representa um impeditivo na utilização desses dados para a exemplificação da metodologia apresentada.

O modelo [1.1] constitui a base deste trabalho, que tem como objetivo discutir a construção de curvas de referência em situações nas quais a relação entre a variável de interesse e a preditora é intrinsecamente não-linear além de obter intervalos de confiança para essas curvas.

No capítulo 2, apresentam-se detalhes sobre os modelos não linearizáveis de interesse e discute-se uma estratégia para a construção das curvas de referência e de seus respectivos intervalos de confiança. No capítulo 3, apresenta-se uma aplicação prática da estratégia proposta no capítulo 2. Finalmente no capítulo 4, discutem-se os resultados e fazem-se sugestões para futuras pesquisas. 


\section{Capítulo 2}

\section{Modelos Não Linearizáveis para Dados Longitudinais}

Os modelos de interesse neste trabalho são modelos de curvas de crescimento que podem ser escritos para o indivíduo $\mathrm{i}(\mathrm{i}=1, \ldots, \mathrm{n})$, como

$$
\mathbf{y}_{\mathrm{i}}=\mathbf{f}\left(\mathbf{X}_{\mathrm{i}}, \boldsymbol{\mu}, \mathbf{a}_{\mathrm{i}}\right)+\mathbf{e}_{\mathrm{i}}
$$

em que $\mathbf{y}_{\mathrm{i}}\left(\mathrm{p}_{\mathrm{i}} \mathrm{x} 1\right)$ é o perfil de respostas, $\mathbf{X}_{\mathrm{i}}\left(\mathrm{p}_{\mathrm{i}} \mathrm{xm}\right)$ é uma matriz com os valores de $\mathrm{m}$ variáveis preditoras, $\mathbf{f}\left(\mathrm{p}_{\mathrm{i}} \mathrm{x} 1\right)$ é um vetor com os valores de uma função que descreve a curva de crescimento, $\boldsymbol{\mu}$ (bx1) é um vetor de efeitos fixos (parâmetros populacionais), $\mathbf{a}_{\mathbf{i}}(\mathrm{bx} 1)$ é um vetor de efeitos aleatórios, e $\mathbf{e}_{\mathrm{i}}\left(\mathrm{p}_{\mathrm{i}} \mathrm{x} 1\right)$ é um vetor de erros aleatórios. Além disso, assume-se que $\mathbf{f}\left(\mathbf{X}_{\mathrm{i}}, \boldsymbol{\mu}, \mathbf{a}_{\mathrm{i}}\right)$ com $\Delta(\mathrm{bxb})$ representando uma matriz simétrica definida positiva não estruturada, $\mathbf{e}_{\mathrm{i}} \sim N_{\mathrm{p}_{\mathrm{i}}}\left(\mathbf{0}, \sigma^{2} \mathbf{I}_{\mathrm{p}_{\mathrm{i}}}\right)$ com $\mathbf{I}_{\mathrm{p}_{\mathrm{i}}}\left(\mathrm{p}_{\mathrm{i}} \mathrm{xp} \mathrm{p}_{\mathrm{i}}\right)$ denotando uma matriz identidade e $\mathbf{a}_{\mathrm{i}}$ e $\mathbf{e}_{\mathrm{i}}$ são independentes entre si. Considerando o modelo [2.1], tem-se

$$
\mathbf{y}_{\mathrm{i}} \mid \mathbf{a}_{\mathrm{i}} \sim N_{\mathrm{p}_{\mathrm{i}}}\left(\mathbf{f}\left(\mathbf{X}_{\mathrm{i}}, \boldsymbol{\mu}, \mathbf{a}_{\mathrm{i}}\right), \sigma^{2} \mathbf{I}_{\mathbf{p}_{\mathrm{i}}}\right) .
$$

Apesar de se poder especificar uma expressão analítica para a função densidade de probabilidade de $\mathbf{y}_{\mathrm{i}} \mid \mathbf{a}_{\mathrm{i}}$, em geral não há expressão analítica para a função densidade de probabilidade de $\mathbf{y}_{\mathbf{i}}$ (Davidian and Giltinan, 1998). 


\section{a. Especificação do modelo aproximado}

No sentido de se obter expressão analítica para a função densidade de probabilidade de $\mathbf{y}_{\mathrm{i}}$, um modelo aproximado para $\mathbf{y}_{i}$ é especificado como em Ogliari and Andrade (2001). Esse modelo é obtido por uma expansão de Taylor de primeira ordem de $\mathbf{f}\left(\mathbf{X}_{\mathrm{i}}, \boldsymbol{\mu}, \mathbf{a}_{\mathrm{i}}\right)$ em torno do ponto $\mathbf{a}_{\mathrm{i}}=\mathbf{0}$ e pode ser escrito como

$$
\mathbf{y}_{\mathrm{i}} \approx \mathbf{f}\left(\mathbf{X}_{\mathrm{i}}, \boldsymbol{\mu}, \mathbf{0}\right)+\mathbf{Z}_{\mathrm{i}}(\boldsymbol{\mu}) \mathbf{a}_{\mathrm{i}}+\mathbf{e}_{\mathrm{i}}
$$

com

$$
\mathbf{Z}_{\mathrm{i}}(\boldsymbol{\mu})=\left[\partial \mathbf{f}\left(\mathbf{X}_{\mathrm{i}}, \boldsymbol{\mu}, \mathbf{a}_{\mathrm{i}}\right) / \partial \mathbf{a}_{\mathrm{i}}^{\mathrm{t}}\right]_{\mathbf{a}_{\mathrm{i}}=\mathbf{0}} .
$$

Como consequêencia, tem-se um modelo "linear" em relação aos efeitos aleatórios, em que: (a) a distribuição de $\mathbf{y}_{\mathrm{i}}$ depende da matriz $\mathbf{X}_{\mathrm{i}}$ através de $\mathbf{f}\left(\mathbf{X}_{\mathrm{i}}, \boldsymbol{\mu}, \mathbf{0}\right)$ e da derivada de $\mathbf{f}\left(\mathbf{X}_{\mathrm{i}}, \boldsymbol{\mu}, \mathbf{a}_{\mathbf{i}}\right)$; (b) $\operatorname{Var}\left[\mathbf{y}_{\mathrm{i}}\right]$ depende dos efeitos fixos através da matriz $\mathbf{Z}_{i}(\boldsymbol{\mu})$; (c) o modelo apresenta $[2 b+b(b-1) / 2+1]$ parâmetros a serem estimados, a saber, $\mathrm{b}$ efeitos fixos em $\boldsymbol{\mu}$, b parâmetros de variância em $\Delta, \mathrm{b}(\mathrm{b}-1) / 2$ parâmetros de covariância em $\Delta$ e o parâmetro $\sigma^{2}$. Então

$$
\begin{aligned}
& \mathbf{y}_{\mathrm{i}} \mid \mathbf{a}_{\mathrm{i}} \sim N_{\mathrm{p}_{\mathrm{i}}}\left(\mathbf{f}\left(\mathbf{X}_{\mathrm{i}}, \boldsymbol{\mu}, \mathbf{0}\right)+\mathbf{Z}_{\mathrm{i}}(\boldsymbol{\mu}) \mathbf{a}_{\mathrm{i}}, \sigma^{2} \mathbf{I}_{\mathrm{p}_{\mathrm{i}}}\right) \mathrm{e} \\
& \mathbf{y}_{\mathrm{i}} \sim N_{\mathrm{p}_{\mathrm{i}}}\left(\mathbf{f}\left(\mathbf{X}_{\mathrm{i}}, \boldsymbol{\mu}, \mathbf{0}\right), \mathbf{Z}_{\mathrm{i}}(\boldsymbol{\mu}) \Delta \mathbf{Z}_{\mathrm{i}}(\boldsymbol{\mu})^{\mathrm{t}}+\sigma^{2} \mathbf{I}_{\mathrm{p}_{\mathrm{i}}}\right) .
\end{aligned}
$$

Estimativas para os respectivos parâmetros do modelo aproximado [2.2] podem ser obtidas por máxima verossimilhança. O leitor pode consultar Singer and Andrade (2000) para maiores detalhes. 


\section{b. Estimação por máxima verossimilhança}

Seja $\omega([b+b(b-1) / 2+1] x 1)$ o vetor dos parâmetros da estrutura de covariância dos dados. Estimadores de $\boldsymbol{\mu}$ e $\boldsymbol{\omega}$ (digamos $\hat{\boldsymbol{\mu}}$ e $\hat{\boldsymbol{\omega}}$ ) podem ser obtidos por meio da maximização da log-verossimilhança

$$
\begin{aligned}
& L\left(\boldsymbol{\mu}, \boldsymbol{\omega} \mid \mathbf{y}_{\mathrm{i}}, \ldots, \mathbf{y}_{\mathrm{n}}\right)=\left(\frac{1}{2}\right) \log (2 \pi) \sum_{\mathrm{i}=1}^{\mathrm{n}} \mathrm{p}_{\mathrm{i}}-\left(\frac{1}{2}\right) \sum_{\mathrm{i}=1}^{\mathrm{n}} \log \left|\mathbf{Z}_{\mathrm{i}}(\boldsymbol{\mu}) \Delta \mathbf{Z}_{\mathrm{i}}(\boldsymbol{\mu})^{\mathrm{t}}+\boldsymbol{\sigma}^{2} \mathbf{I}_{\mathrm{p}_{\mathrm{i}}}\right| \\
& -\left(\frac{1}{2}\right) \sum_{\mathrm{i}=1}^{\mathrm{n}}\left[\mathbf{y}_{\mathrm{i}}-\mathbf{f}\left(\mathbf{X}_{\mathrm{i}}, \boldsymbol{\mu}, \mathbf{0}\right)\right]^{\mathrm{t}}\left(\mathbf{Z}_{\mathrm{i}}(\boldsymbol{\mu}) \Delta \mathbf{Z}_{\mathrm{i}}(\boldsymbol{\mu})^{\mathrm{t}}+\boldsymbol{\sigma}^{2} \mathbf{I}_{\mathrm{p}_{\mathrm{i}}}\right)^{-1}\left[\mathbf{y}_{\mathrm{i}}-\mathbf{f}\left(\mathbf{X}_{\mathrm{i}}, \boldsymbol{\mu}, \mathbf{0}\right)\right] .
\end{aligned}
$$

Quando o modelo [2.2] é uma boa aproximação para [2.1], o tamanho da amostra é suficientemente grande e algumas condições de regularidade para a matriz $\mathbf{X}_{i}$ estão satisfeitas, os estimadores de $\boldsymbol{\mu}$ e $\boldsymbol{\omega}$ obtidos pela maximização de $L\left(\boldsymbol{\mu}, \boldsymbol{\omega} \mid \mathbf{y}_{\mathrm{i}}, \ldots, \mathbf{y}_{\mathrm{n}}\right)$ são também estimadores de máxima verossimilhança dos parâmetros do modelo [2.1] (Davidian and Giltinan, 1998). Estes estimadores apresentam uma série de propriedades assintóticas ótimas que podem ser utilizadas para fazer inferências para $\boldsymbol{\mu}$ e $\boldsymbol{\omega}$ e/ou funções das mesmas (Sen and Singer, 1993).

\section{c. Tipos de curvas de referência}

Pode-se considerar dois possíveis tipos de curvas de referência: (i) curvas de referência para a identificação de valores extremos e/ou inesperados, (ii) curvas de referência para a identificação de perfis individuais extremos e/ou inesperados. 
O primeiro corresponde ao tipo de curva considerado por Royston (1995) e Sañudo (2000) e tem como objeto de análise uma única observação entre as diversas tomadas no i-ésimo indivíduo. Este tipo de curva de referência considera toda a variabilidade observada no conjunto de dados para sua construção.

O segundo tipo de curva de referência tem como objeto de análise o perfil individual ajustado ao conjunto de observações tomadas no i-ésimo indivíduo. Para a análise deste perfil individual ajustado consideram-se somente as variabilidades entre e intra-indivíduo, modeladas por $\mathbf{f}\left(\mathbf{X}_{\mathrm{i}}, \boldsymbol{\mu}, \mathbf{a}_{\mathbf{i}}\right)$, sendo desprezada a variabilidade presente em $\mathbf{e}_{\mathrm{i}}$.

Para a construção de ambos os tipos de curva de referência, apresentam-se propostas de abordagem análogas à utilizada por Royston (1995). A diferença está na consideração ou não do erro aleatório no cálculo das curvas de referência.

\section{d. Curvas de referência para identificação de valores extremos}

Considerando o modelo aproximado [2.2], pode-se utilizar o modelo probabilístico normal para construir curvas de referência aproximadas. Estas curvas são obtidas através de

$$
\mathbf{y}_{\gamma}=\mathbf{f}\left(\mathbf{X}_{\mathrm{i}}, \boldsymbol{\mu}, \mathbf{0}\right)+z_{\boldsymbol{\gamma}} \mathbf{D P}\left(\mathbf{Z}_{\mathrm{i}}(\boldsymbol{\mu}) \Delta \mathbf{Z}_{\mathrm{i}}(\boldsymbol{\mu})^{\mathrm{t}}+\sigma^{2} \mathbf{I}_{\mathrm{p}_{\mathrm{i}}}\right)
$$

em que $\mathbf{y}_{\gamma}\left(\mathrm{p}_{\mathrm{i}} \mathrm{x} 1\right)$ é o vetor com os valores da curva de referência de $100 \gamma \%$ $(0<\gamma<1), \operatorname{DP}\left(\mathbf{Z}_{\mathrm{i}}(\boldsymbol{\mu}) \Delta \mathbf{Z}_{\mathrm{i}}(\boldsymbol{\mu})^{\mathrm{t}}+\sigma^{2} \mathbf{I}_{\mathrm{p}_{\mathrm{i}}}\right)\left(\mathrm{p}_{\mathrm{i}} \times 1\right)$ é um vetor, cujos elementos são as raízes quadradas dos elementos da diagonal de $\boldsymbol{Z}_{\mathrm{i}}(\boldsymbol{\mu}) \Delta \boldsymbol{Z}_{\mathrm{i}}(\boldsymbol{\mu})^{\mathrm{t}}+\sigma^{2} \mathbf{I}_{\mathrm{p}_{\mathrm{i}}}$ e $\mathrm{Z}_{\gamma}$ é o 
percentil $100 \gamma \%$ da distribuição normal padrão. Variando $\gamma$ pode-se construir as curvas de referência associadas aos vários percentis populacionais de interesse.

O estimador de máxima verossimilhança para a curva de referência pode ser obtido com a substituição dos parâmetros $\boldsymbol{\mu}$ e $\boldsymbol{\omega}$ em [2.3] por $\hat{\boldsymbol{\mu}}$ e $\hat{\boldsymbol{\omega}}$. Os limites inferior e superior correspondentes aos intervalos de confiança assintóticos com coeficiente de confiança de $100(1-\lambda) \%(0<\lambda<1)$ para os elementos de $\widehat{\mathbf{y}_{\gamma}}$ podem ser calculados por meio de

$$
\begin{aligned}
\mathrm{LS}_{1-\lambda} & =\widehat{\mathbf{y}_{\gamma}}+z_{1-\lambda / 2} \operatorname{DP}\left(\operatorname{Var}\left[\widehat{\mathbf{y}_{\gamma}}\right]\right) \\
\mathrm{LI}_{1-\lambda} & =\widehat{\mathbf{y}_{\gamma}}+z_{\lambda / 2} \operatorname{DP}\left(\operatorname{Var}\left[\widehat{\mathbf{y}_{\gamma}}\right]\right)
\end{aligned}
$$

em que $z_{\zeta}$ é o quantil de ordem $100 \zeta \%$ da distribuição normal padrão.

\section{e. Curvas de referência para identificação de perfis individuais extremos}

De forma análoga à construção das curvas de referência para identificação de valores extremos, pode-se considerar o modelo aproximado [2.2] e o modelo probabilístico normal para construir curvas de referência aproximadas para os perfis individuais modelados por $\mathbf{f}\left(\mathbf{X}_{\mathbf{i}}, \boldsymbol{\mu}, \mathbf{a}_{\mathbf{i}}\right)$. Estes perfis são aproximadamente modelados por

$$
\mathbf{f}\left(\mathbf{X}_{\mathrm{i}}, \boldsymbol{\mu}, \mathbf{a}_{\mathrm{i}}\right) \approx \mathbf{f}\left(\mathbf{X}_{\mathrm{i}}, \boldsymbol{\mu}, \mathbf{0}\right)+\mathbf{Z}_{\mathrm{i}}(\boldsymbol{\mu}) \mathbf{a}_{\mathrm{i}}
$$

Então

$$
\mathbf{f}\left(\mathbf{X}_{\mathrm{i}}, \boldsymbol{\mu}, \mathbf{a}_{\mathrm{i}}\right) \stackrel{\mathrm{a}}{\sim} N_{\mathrm{p}_{\mathrm{i}}}\left(\mathbf{f}\left(\mathbf{X}_{\mathrm{i}}, \boldsymbol{\mu}, \mathbf{0}\right), \mathbf{Z}_{\mathrm{i}}(\boldsymbol{\mu}) \Delta \mathbf{Z}_{\mathrm{i}}(\boldsymbol{\mu})^{\mathrm{t}}\right)
$$


As curvas de referência $\mathbf{y}_{\gamma}$ adequadas à identificação de perfis extremos e/ou inesperados são obtidas de

$$
\mathbf{y}_{\gamma}=\mathbf{f}\left(\mathbf{X}_{\mathrm{i}}, \boldsymbol{\mu}, \mathbf{0}\right)+z_{\gamma} \mathbf{D P}\left(\mathbb{Z}_{\mathrm{i}}(\boldsymbol{\mu}) \Delta \mathbf{Z}_{\mathrm{i}}(\boldsymbol{\mu})^{\mathrm{t}}\right)
$$

e são diferentes de [2.3] pela exclusão da variância de $\mathbf{e}_{i}$. A construção dos respectivos intervalos de confiança univariados para cada valor desta curva segue um processo análogo ao descrito na seção anterior.

\section{f. Estratégias de análise}

Para realizarmos o ajuste do modelo [2.2], sugerimos a estratégia de análise, descrita a seguir.

1. Por meio de uma análise descritiva dos dados e de uma investigação da literatura sobre a característica de interesse, obtém-se uma ou mais funções não lineares para a curva de crescimento descrita pelos perfis individuais. Para cada perfil individual, ajustam-se por meio de mínimos quadrados ordinários os modelos não lineares correspondentes às funções escolhidas (Souza, 1998).

2. Possíveis modelos de forma [2.2] são selecionados com diferentes especificações para os efeitos aleatórios. Obtém-se as expressões para a verossimilhança e ajustam-se os modelos por meio de máxima verossimilhança.

3. Para avaliar o ajuste dos modelos, pode-se realizar a análise dos resíduos de cada um além de uma comparação grosseira entre os preditores para os perfis individuais obtidos sob os modelos ajustados por meio de mínimos quadrados ordinários e os preditores obtidos sob o modelo [2.2]. São 
desprezados os modelos cuja análise dos resíduos e dos preditores indiquem falta de ajuste e dentre os demais modelos, o modelo final pode ser escolhido por meio da comparação do Critério da Informação de Akaike (AIC) e/ou do Critério da Informação Bayesiano de Schwarz (BIC) [ver Jones (1993), por exemplo].

\section{g. Especificação para o caso da variação do $\mathrm{VO}_{2}$}

Sob o modelo exponencial [1.1], pode-se especificar para o atleta i $(\mathrm{i}=1, \ldots, 22)$ o modelo aproximado

$$
\mathbf{y}_{\mathrm{i}}=\mathbf{f}\left(\mathbf{t}_{\mathrm{i}}, \boldsymbol{\mu}, \mathbf{0}\right)+\mathbf{Z}_{\mathrm{i}}(\boldsymbol{\mu}) \mathbf{a}_{\mathrm{i}}+\mathbf{e}_{\mathrm{i}},
$$

em que $\mathbf{y}_{\mathrm{i}}\left(\mathrm{p}_{\mathrm{i}} \mathrm{x} 1\right)$ é o vetor das observações de $\Delta \mathrm{VO}_{2}$ para os momentos em que foram realizadas observações, $\mathbf{f}\left(\mathbf{t}_{\mathrm{i}}, \boldsymbol{\mu}, \mathbf{0}\right)\left(\mathrm{p}_{\mathrm{i}} \mathrm{x} 1\right)$ é o vetor cujos elementos são

$$
f\left(\mathrm{t}, \boldsymbol{\mu}, \mathbf{a}_{\mathrm{i}}\right)=\left(\beta+\mathrm{b}_{\mathrm{i}}\right) \cdot\left\{1-\exp \left[-\frac{\mathrm{t}-\left(\delta+\mathrm{d}_{\mathrm{i}}\right)}{\left(\tau+\mathrm{r}_{\mathrm{i}}\right)}\right]\right\},
$$

$\mathbf{t}_{\mathrm{i}}\left(\mathrm{p}_{\mathrm{i}} \mathrm{x} 1\right)$ é o vetor cujos elementos correspondem aos tempos em que cada observação de $\mathbf{y}_{i}$ foi obtida, $\boldsymbol{\mu}(3 \times 1)$ é o vetor dos parâmetros fixos, $\mathbf{a}_{i}(3 \times 1)$ é o vetor dos efeitos aleatórios, e os três elementos de cada linha de

$$
\mathbf{Z}_{\mathrm{i}}(\boldsymbol{\mu})=\left[\partial \mathbf{f}\left(\mathbf{t}_{\mathrm{i}}, \boldsymbol{\mu}, \mathbf{a}_{\mathrm{i}}\right) / \partial \mathbf{a}_{\mathrm{i}}^{\mathrm{t}}\right]_{\mathbf{a}_{\mathrm{i}}=\mathbf{0}}
$$

são respectivamente

$$
\partial f\left(\mathrm{t}, \boldsymbol{\mu}, \mathbf{a}_{\mathrm{i}}\right) /\left.\partial \mathrm{b}_{\mathrm{i}}\right|_{\mathbf{a}_{\mathrm{i}}=\mathbf{0}}=1-\exp \left[-\frac{(\mathrm{t}-\delta)}{\tau}\right],
$$




$$
\begin{gathered}
\partial f\left(\mathrm{t}, \boldsymbol{\mu}, \mathbf{a}_{i}\right) /\left.\partial \mathrm{d}_{\mathrm{i}}\right|_{\mathbf{a}_{\mathrm{i}}=\mathbf{0}}=-\frac{\beta}{(\mathrm{t}-\delta)} \cdot \exp \left[-\frac{(\mathrm{t}-\delta)}{\tau}\right] \mathrm{e} \\
\partial f\left(\mathrm{t}, \boldsymbol{\mu}, \mathbf{a}_{\mathrm{i}}\right) /\left.\partial \mathrm{r}_{\mathrm{i}}\right|_{\mathbf{a}_{\mathrm{i}}=\mathbf{0}}=-\frac{\beta}{(\mathrm{t}-\delta)} \cdot \exp \left[-\frac{(\mathrm{t}-\delta)}{\tau}\right] \cdot \frac{1}{\tau^{2}} .
\end{gathered}
$$

Sob este modelo, $\boldsymbol{\mu}=(\beta, \delta, \tau)^{\mathrm{t}}$ e $\boldsymbol{\omega}=\left(\sigma_{\mathrm{b}}^{2}, \sigma_{\mathrm{d}}^{2}, \sigma_{\mathrm{r}}^{2}, \sigma_{\mathrm{bd}}, \sigma_{\mathrm{br}}, \sigma_{\mathrm{dr}}, \sigma^{2}\right)^{\mathrm{t}}$.

$\mathrm{O}$ ajuste do modelo [2.5] e sua avaliação podem ser realizados por meio da estratégia sugerida na seção anterior. Para cada perfil individual, ajusta-se de forma exploratória o modelo [1.1] por meio de mínimos quadrados ordinários. $\mathrm{O}$ ajuste do modelo [2.2] é feito por meio de máxima verossimilhança utilizando um método iterativo. Posteriormente realiza-se uma análise dos resíduos e uma comparação dos preditores obtidos por meio desses modelos. No próximo capítulo, exemplificamos este processo para uma possível especificação do modelo [2.5]. 


\section{Capítulo 3}

\section{Análise dos Dados}

Neste capítulo ilustra-se a metodologia apresentada por meio do ajuste de curvas de referência para a variação de $\mathrm{VO}_{2}$ ao longo do tempo com os dados do estudo descrito no Capítulo 1.

\section{a. Análise preliminar dos dados}

Os dados consistem de 1858 observações tomadas em 22 atletas, após os 20 segundos iniciais da corrida. A Figura 3.1 apresenta os perfis individuais de $\Delta \mathrm{VO}_{2}$ de todos os atletas. Nessa figura, pode-se ver que os valores de $\Delta \mathrm{VO}_{2}$ obedecem a um mesmo padrão temporal, com um período de crescimento no início e posterior estabilização. Este padrão pode ser melhor visualizado na Figura $3.2 \mathrm{em}$ que apenas os 180 segundos iniciais são incluídos. Nota-se uma grande variabilidade entre indivíduos, com os valores de $\Delta \mathrm{VO}_{2}$ variando de $200 \mathrm{ml} / \mathrm{min}$ negativos (representando um decréscimo em relação ao nível basal) até $3000 \mathrm{ml} / \mathrm{min}$. Nas Figuras 3.1 e 3.2, também pode-se notar um certo "paralelismo" entre os perfis individuais, o que sugere uma correlação não nula entre os dados observados no mesmo atleta. 
Figura 3.1. Perfis individuais de variação do consumo de oxigênio em relação aos valores basais.

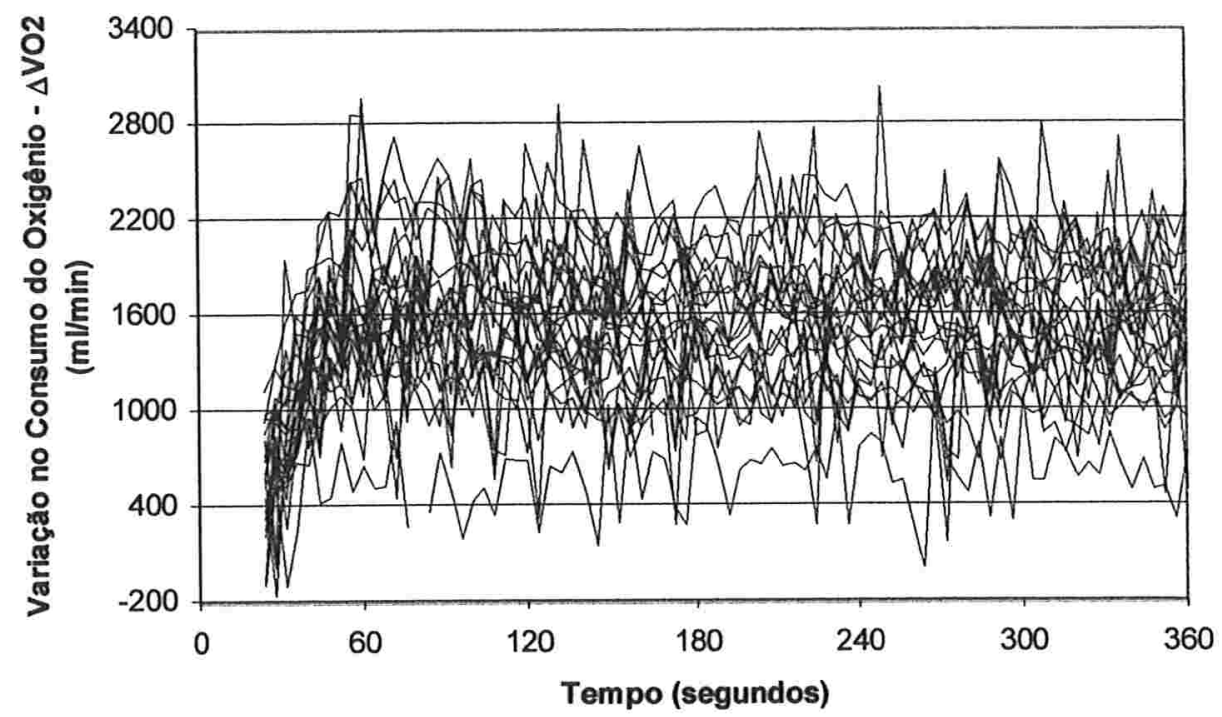

Figura 3.2. Perfis individuais de variação do consumo de oxigênio em relação aos valores basais nos primeiros 180 segundos.

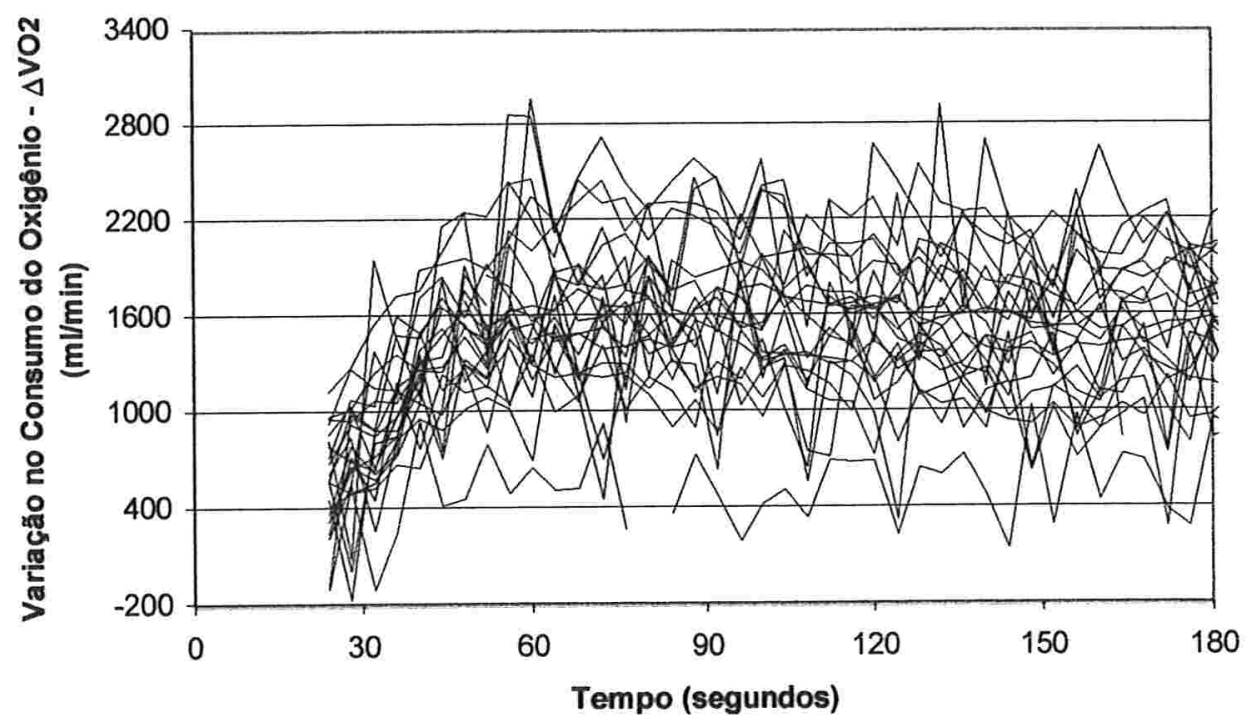


Na Tabela 3.1 estão apresentadas a média, a mediana, o desvio padrão, o primeiro e terceiro quartis e o coeficiente de variação dos $\Delta \mathrm{VO}_{2}$, em cada momento de avaliação. As médias e as medianas em cada momento são semelhantes, sugerindo uma simetria na distribuição dos dados. Esse aspecto pode ser visualizado na Figura $3.3 \mathrm{em}$ que se representam os perfis das médias, dos quartis, dos mínimos e dos máximos. Além disso, os perfis das médias, das medianas, dos quartis, dos mínimos e dos máximos parecem seguir o padrão descrito na literatura [Fukuba et al. (2002) e Rossiter et al. (2001), por exemplo] e apresentado na Figura 1.2.

Na Figura 3.4, apresentam-se gráficos do tipo "box-plot" para alguns dos momentos de monitoramento (12 em 12 segundos). Há indícios de heterocedasticidade, pois o período de estabilização parece apresentar uma variabilidade entre indivíduos diferente da variabilidade observada no período de crescimento. Apesar disso, a figura reforça a impressão de simetria na distribuição dos valores de $\Delta \mathrm{VO}_{2}$, além de sugerir poucos valores extremos. Esses aspectos não apresentam evidências contrárias à utilização de distribuições normais como modelos probabilísticos para os $\Delta \mathrm{VO}_{2}$. Para avaliar essa hipótese, utiliza-se o teste de normalidade de Shapiro-Wilk (Neter et al., 1996) para cada um dos 85 momentos considerados. Em 82 desses momentos, a hipótese de que as variações no consumo de oxigênio têm distribuição normal não foi rejeitada $(p>0,05)$. Os níveis descritivos (valores p) obtidos podem ser encontrados na Tabela 3.2.

De forma exploratória, foram ajustados modelos monoexponenciais descritos em [1.1] para o perfil das médias, dos desvios padrões de $\triangle \mathrm{VO} 2$ e para os perfis individuais de cada atleta por mínimos 
Tabela 3.1. Estatísticas descritivas para a variação no consumo de oxigênio - $\Delta \mathrm{VO}_{2}$ $(\mathrm{ml} / \mathrm{min})$.

\begin{tabular}{|c|c|c|c|c|c|c|c|}
\hline Tempo & $\mathbf{N}$ & Média & Mediana & DP & $1^{\circ}$ Quartil & $3^{\circ}$ Quartil & CV \\
\hline 24 & 22 & 551 & 562 & 330 & 324 & 803 & $60 \%$ \\
\hline 28 & 21 & 750 & 778 & 355 & 546 & 1006 & $47 \%$ \\
\hline 32 & 22 & 839 & 824 & 445 & 582 & 1060 & $53 \%$ \\
\hline 36 & 22 & 1037 & 1010 & 344 & 826 & 1169 & $33 \%$ \\
\hline 40 & 22 & 1259 & 1257 & 307 & 1063 & 1441 & $24 \%$ \\
\hline 44 & 21 & 1367 & 1338 & 470 & 1023 & 1709 & $34 \%$ \\
\hline 48 & 21 & 1517 & 1524 & 419 & 1292 & 1752 & $28 \%$ \\
\hline 52 & 22 & 1412 & 1392 & 344 & 1199 & 1523 & $24 \%$ \\
\hline 56 & 21 & 1667 & 1621 & 543 & 1446 & 2045 & $33 \%$ \\
\hline 60 & 22 & 1645 & 1526 & 609 & 1300 & 1953 & $37 \%$ \\
\hline 64 & 22 & 1624 & 1627 & 419 & 1441 & 1888 & $26 \%$ \\
\hline 68 & 22 & 1560 & 1525 & 485 & 1229 & 1799 & $31 \%$ \\
\hline 72 & 22 & 1619 & 1622 & 535 & 1395 & 1825 & $33 \%$ \\
\hline 76 & 22 & 1543 & 1591 & 502 & 1250 & 1784 & $33 \%$ \\
\hline 80 & 21 & 1707 & 1719 & 382 & 1444 & 1966 & $22 \%$ \\
\hline 84 & 22 & 1536 & 1511 & 464 & 1334 & 1738 & $30 \%$ \\
\hline 88 & 21 & 1631 & 1587 & 522 & 1283 & 1830 & $32 \%$ \\
\hline 92 & 21 & 1554 & 1569 & 643 & 1205 & 1878 & $41 \%$ \\
\hline 96 & 22 & 1608 & 1733 & 458 & 1436 & 1920 & $28 \%$ \\
\hline 100 & 22 & 1611 & 1503 & 538 & 1273 & 1959 & $33 \%$ \\
\hline 104 & 22 & 1623 & 1672 & 453 & 1344 & 1776 & $28 \%$ \\
\hline 108 & 21 & 1402 & 1359 & 522 & 1147 & 1834 & $37 \%$ \\
\hline 112 & 22 & 1584 & 1653 & 448 & 1310 & 1905 & $28 \%$ \\
\hline 116 & 22 & 1534 & 1574 & 363 & 1325 & 1729 & $24 \%$ \\
\hline 120 & 22 & 1553 & 1595 & 496 & 1195 & 1828 & $32 \%$ \\
\hline 124 & 22 & 1497 & 1511 & 548 & 1313 & 1820 & $37 \%$ \\
\hline 128 & 22 & 1544 & 1498 & 424 & 1331 & 1695 & $27 \%$ \\
\hline 132 & 22 & 1591 & 1550 & 503 & 1300 & 1884 & $32 \%$ \\
\hline 136 & 22 & 1528 & 1535 & 428 & 1214 & 1820 & $28 \%$ \\
\hline 140 & 22 & 1491 & 1458 & 502 & 1167 & 1767 & $34 \%$ \\
\hline 144 & 22 & 1492 & 1518 & 488 & 1200 & 1781 & $33 \%$ \\
\hline 148 & 22 & 1504 & 1547 & 440 & 1239 & 1803 & $29 \%$ \\
\hline 152 & 22 & 1423 & 1443 & 394 & 1229 & 1679 & $28 \%$ \\
\hline 156 & 22 & 1495 & 1476 & 507 & 1064 & 1908 & $34 \%$ \\
\hline 160 & 22 & 1448 & 1464 & 496 & 1076 & 1809 & $34 \%$ \\
\hline 164 & 22 & 1508 & 1569 & 423 & 1191 & 1823 & $28 \%$ \\
\hline 168 & 21 & 1555 & 1527 & 398 & 1343 & 1819 & $26 \%$ \\
\hline 172 & 21 & 1466 & 1483 & 605 & 1182 & 1939 & $41 \%$ \\
\hline 176 & 22 & 1527 & 1592 & 455 & 1280 & 1891 & $30 \%$ \\
\hline 180 & 22 & 1571 & 1576 & 366 & 1426 & 1786 & $23 \%$ \\
\hline 184 & 22 & 1520 & 1526 & 410 & 1162 & 1812 & $27 \%$ \\
\hline 188 & 22 & 1521 & 1521 & 425 & 1290 & 1769 & $28 \%$ \\
\hline 192 & 22 & 1454 & 1396 & 450 & 1142 & 1757 & $31 \%$ \\
\hline 196 & 22 & 1473 & 1480 & 396 & 1240 & 1725 & $27 \%$ \\
\hline 200 & 22 & 1488 & 1557 & 405 & 1267 & 1744 & $27 \%$ \\
\hline 204 & 22 & 1589 & 1457 & 497 & 1246 & 1919 & $31 \%$ \\
\hline 208 & 22 & 1555 & 1643 & 476 & 1119 & 1876 & $31 \%$ \\
\hline 212 & 22 & 1539 & 1585 & 418 & 1261 & 1731 & $27 \%$ \\
\hline 216 & 22 & 1550 & 1572 & 419 & 1253 & 1785 & $27 \%$ \\
\hline 220 & 22 & 1567 & 1585 & 463 & 1367 & 1821 & $30 \%$ \\
\hline 224 & 22 & 1492 & 1549 & 597 & 1203 & 1680 & $40 \%$ \\
\hline 228 & 22 & 1555 & 1523 & 409 & 1331 & 1698 & $26 \%$ \\
\hline
\end{tabular}


Tabela 3.1. (cont.) Estatísticas descritivas para a variação no consumo de oxigênio $\Delta \mathrm{VO}_{2}(\mathrm{ml} / \mathrm{min})$.

\begin{tabular}{|c|c|c|c|c|c|c|c|}
\hline Tempo & $\mathbf{N}$ & Média & Mediana & DP & $1^{\circ}$ Quartil & $3^{\circ}$ Quartil & CV \\
\hline 232 & 22 & 1584 & 1596 & 450 & 1242 & 1883 & $28 \%$ \\
\hline 236 & 22 & 1545 & 1580 & 498 & 1347 & 1855 & $32 \%$ \\
\hline 240 & 22 & 1594 & 1640 & 404 & 1269 & 1926 & $25 \%$ \\
\hline 244 & 22 & 1465 & 1446 & 336 & 1171 & 1701 & $23 \%$ \\
\hline 248 & 22 & 1591 & 1573 & 526 & 1268 & 1796 & $33 \%$ \\
\hline 252 & 22 & 1500 & 1516 & 430 & 1269 & 1754 & $29 \%$ \\
\hline 256 & 22 & 1499 & 1482 & 424 & 1260 & 1860 & $28 \%$ \\
\hline 260 & 22 & 1504 & 1506 & 434 & 1230 & 1766 & $29 \%$ \\
\hline 264 & 22 & 1522 & 1668 & 483 & 1262 & 1801 & $32 \%$ \\
\hline 268 & 22 & 1579 & 1591 & 404 & 1217 & 1858 & $26 \%$ \\
\hline 272 & 22 & 1441 & 1513 & 560 & 1209 & 1822 & $39 \%$ \\
\hline 276 & 22 & 1523 & 1576 & 403 & 1368 & 1752 & $26 \%$ \\
\hline 280 & 22 & 1587 & 1495 & 473 & 1369 & 1918 & $30 \%$ \\
\hline 284 & 21 & 1534 & 1529 & 373 & 1365 & 1790 & $24 \%$ \\
\hline 288 & 22 & 1499 & 1532 & 494 & 1159 & 1916 & $33 \%$ \\
\hline 292 & 22 & 1544 & 1590 & 505 & 1170 & 1785 & $33 \%$ \\
\hline 296 & 21 & 1529 & 1489 & 440 & 1289 & 1765 & $29 \%$ \\
\hline 300 & 22 & 1561 & 1474 & 351 & 1356 & 1882 & $22 \%$ \\
\hline 304 & 22 & 1511 & 1434 & 441 & 1210 & 1839 & $29 \%$ \\
\hline 308 & 22 & 1543 & 1530 & 490 & 1270 & 1792 & $32 \%$ \\
\hline 312 & 22 & 1530 & 1514 & 368 & 1316 & 1790 & $24 \%$ \\
\hline 316 & 22 & 1578 & 1596 & 480 & 1289 & 2008 & $30 \%$ \\
\hline 320 & 22 & 1525 & 1561 & 491 & 1171 & 1945 & $32 \%$ \\
\hline 324 & 22 & 1469 & 1434 & 382 & 1278 & 1828 & $26 \%$ \\
\hline 328 & 22 & 1542 & 1627 & 416 & 1316 & 1811 & $27 \%$ \\
\hline 332 & 22 & 1479 & 1381 & 434 & 1199 & 1782 & $29 \%$ \\
\hline 336 & 22 & 1553 & 1562 & 454 & 1164 & 1812 & $29 \%$ \\
\hline 340 & 22 & 1486 & 1516 & 397 & 1143 & 1693 & $27 \%$ \\
\hline 344 & 22 & 1487 & 1482 & 360 & 1251 & 1758 & $24 \%$ \\
\hline 348 & 22 & 1555 & 1568 & 438 & 1273 & 1873 & $28 \%$ \\
\hline 352 & 22 & 1464 & 1482 & 502 & 1192 & 1828 & $34 \%$ \\
\hline 356 & 22 & 1423 & 1489 & 393 & 1213 & 1660 & $28 \%$ \\
\hline 360 & 22 & 1526 & 1465 & 471 & 1345 & 1764 & $31 \%$ \\
\hline
\end{tabular}


Figura 3.3. Perfis das médias, dos quartis, dos mínimos e dos máximos de cada momento para a variação no consumo de oxigênio em relação aos valores basais.

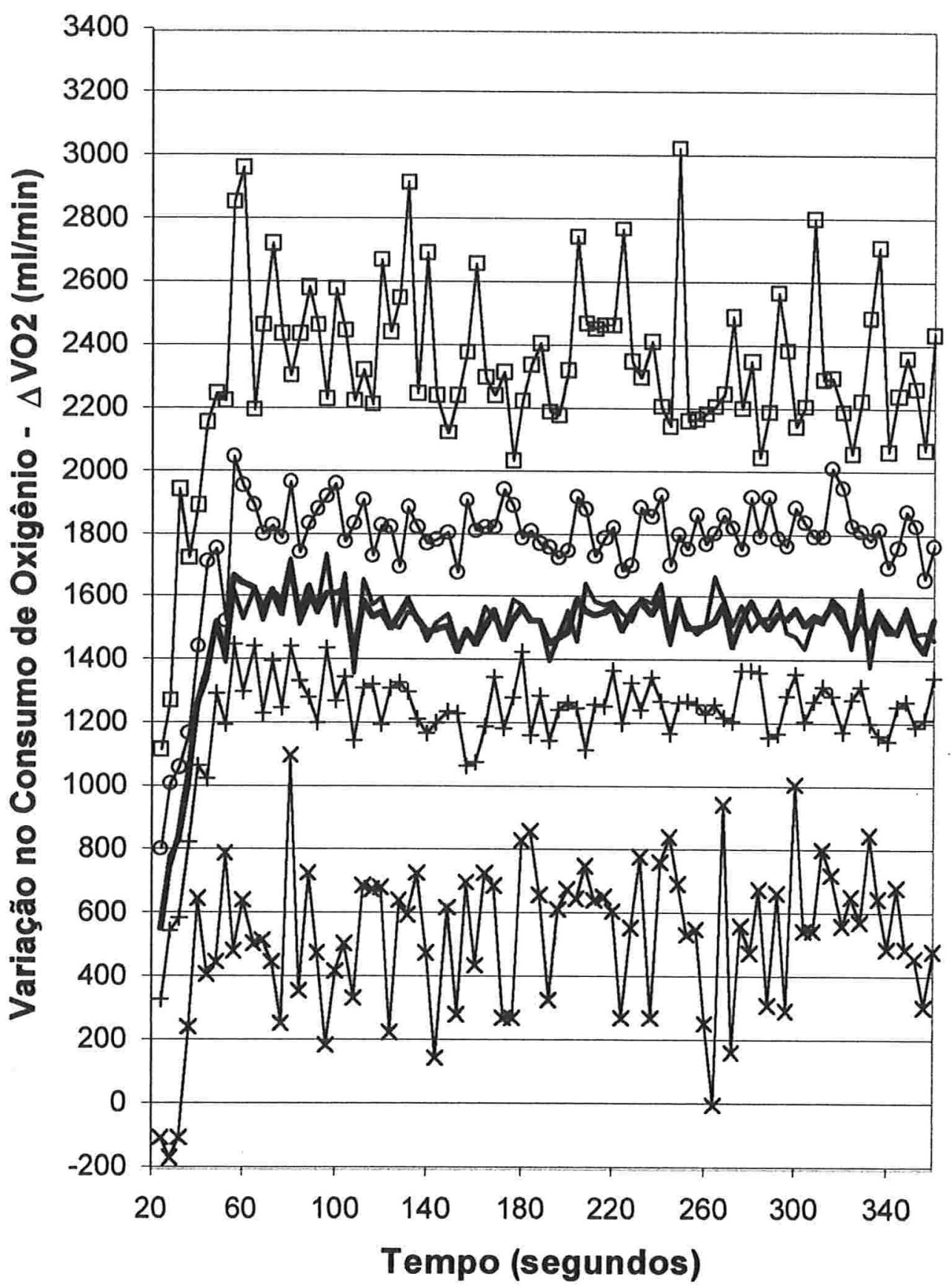

$*$ Mínimo $\longrightarrow 1^{\circ}$ Quartil $\longrightarrow$ Média $\longrightarrow$ Mediana $-3^{\circ}$ Quartil —-Máximo 
Figura 3.4. Boxplots da variação no consumo de oxigênio em relação aos valores basais ( $\mathrm{ml} / \mathrm{min}$ ) para alguns momentos de monitoramento.

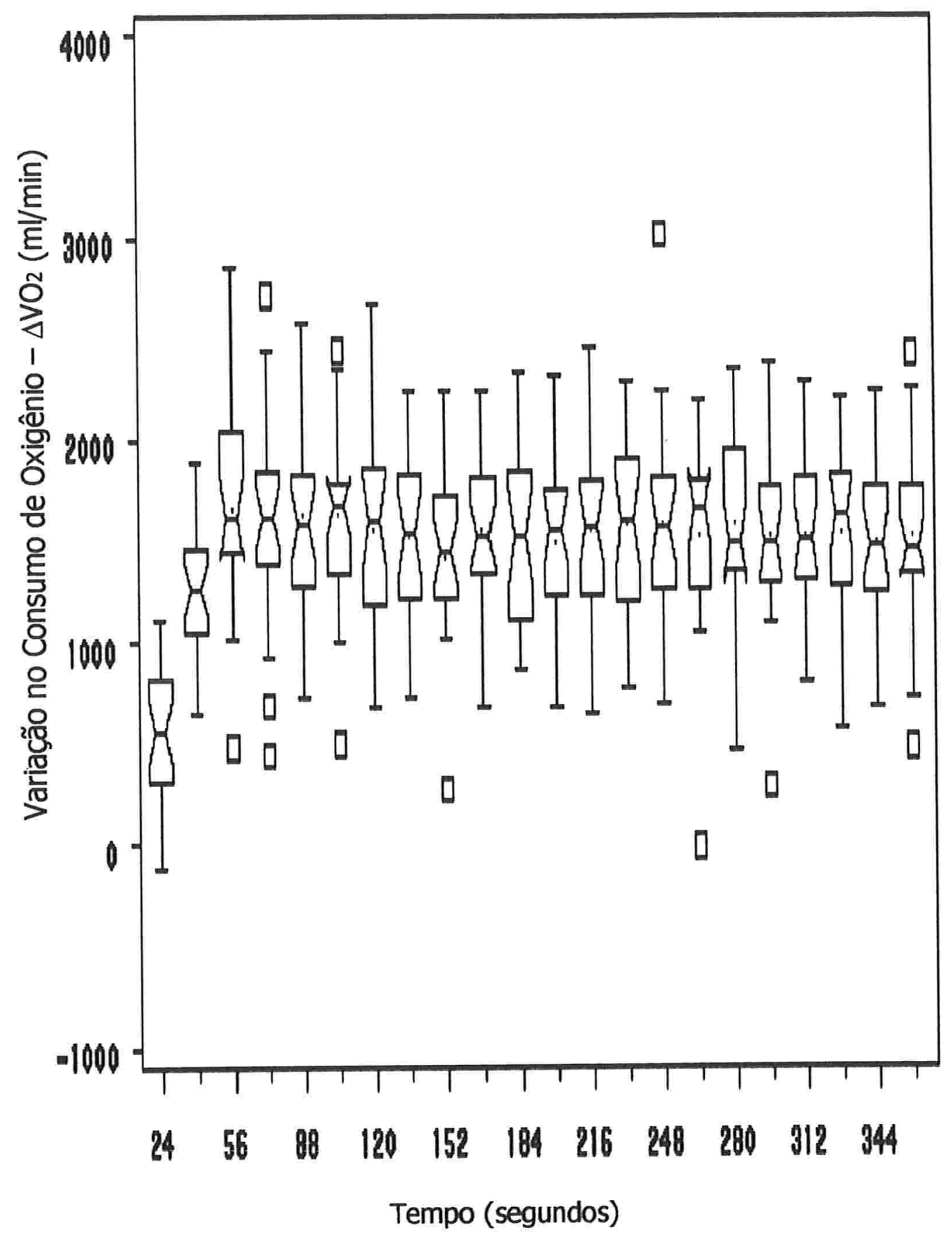


quadrados ordinários. Na Figura 3.5, apresentam-se o perfil das médias, os limites inferior e superior para os respectivos intervalos de confiança com coeficiente de confiança de $95 \%$ para as médias além do perfil dos valores preditos pelo modelo. $\mathrm{O}$ modelo teórico parece adequado para explicar o perfil das médias, apresentando apenas um valor predito não contido no intervalo de confiança para a média, aos 80 segundos de corrida.

Na Figura 3.6, comparam-se os perfis individuais observados para cada atleta com seus respectivos perfis ajustados segundo o modelo [1.1]. Este modelo parece explicar o perfil individual descrito pelas observações. Estes perfis individuais sugerem uma maior variabilidade no nível de $\Delta \mathrm{VO}_{2}$ em que o consumo de cada indivíduo parece se estabilizar do que diferenças entre os momentos de estabilização do nível de $\Delta \mathrm{VO}_{2}$. Por exemplo, para o atleta 25 a estimativa de mínimos quadrados ordinários para o nível em que a variação do consumo de oxigênio se estabiliza é $1157 \mathrm{ml} / \mathrm{min}$ enquanto que o atleta 29 apresenta um nível de estabilização da variação do consumo de oxigênio estimado em 2079 $\mathrm{ml} / \mathrm{min}$.

$\mathrm{Na}$ construção de um modelo para explicar a variabilidade dos valores de $\Delta \mathrm{VO}_{2}$, deve-se levar em conta que: (i) existem várias medidas de $\Delta \mathrm{VO}_{2}$ tomadas no mesmo indivíduo ao longo do tempo; (ii) a relação entre $\Delta \mathrm{VO}_{2}$ e o tempo é explicada por um modelo não linearizável; (iii) os perfis individuais parecem ser explicados por um mesmo tipo de modelo; (iv) a variabilidade entre indivíduos não parece ser constante ao longo do tempo, podendo ser explicada ou por uma função da média ou por uma função mono-exponencial do tempo; (v) o modelo probabilístico normal pode ser utilizado para modelar a distribuição dos valores de $\Delta \mathrm{VO}_{2}$ em cada momento; (vi) não temos os mesmos números de observações para todos os atletas. 
Tabela 3.2. Estatísticas do teste de normalidade de Shapiro-Wilk e respectivos níveis descritivos (valor p).

\begin{tabular}{|c|c|c|c|c|c|}
\hline Tempo & Estatística W & Valor p & Tempo & Estatística W & Nível descritivo \\
\hline 24 & 0,972 & 0,756 & 196 & 0,987 & 0,989 \\
\hline 28 & 0,933 & 0,156 & 200 & 0,976 & 0,852 \\
\hline 32 & 0,973 & 0,773 & 204 & 0,972 & 0,749 \\
\hline 36 & 0,970 & 0,719 & 208 & 0,957 & 0,430 \\
\hline 40 & 0,987 & 0,990 & 212 & 0,987 & 0,988 \\
\hline 44 & 0,980 & 0,919 & 216 & 0,984 & 0,968 \\
\hline 48 & 0,968 & 0,691 & 220 & 0,982 & 0,949 \\
\hline 52 & 0,968 & 0,664 & 224 & 0,980 & 0,912 \\
\hline 56 & 0,968 & 0,684 & 228 & 0,964 & 0,578 \\
\hline 60 & 0,939 & 0,189 & 232 & 0,963 & 0,559 \\
\hline 64 & 0,945 & 0,252 & 236 & 0,968 & 0,660 \\
\hline 68 & 0,962 & 0,540 & 240 & 0,964 & 0,563 \\
\hline 72 & 0,976 & 0,849 & 244 & 0,968 & 0,655 \\
\hline 76 & 0,974 & 0,803 & 248 & 0,965 & 0,592 \\
\hline 80 & 0,948 & 0,307 & 252 & 0,968 & 0,673 \\
\hline 84 & 0,953 & 0,360 & 256 & 0,956 & 0,419 \\
\hline 88 & 0,961 & 0,540 & 260 & 0,942 & 0,222 \\
\hline 92 & 0,971 & 0,753 & 264 & 0,894 & 0,023 \\
\hline 96 & 0,900 & 0,030 & 268 & 0,956 & 0,408 \\
\hline 100 & 0,962 & 0,531 & 272 & 0,960 & 0,493 \\
\hline 104 & 0,958 & 0,444 & 276 & 0,940 & 0,200 \\
\hline 108 & 0,958 & 0,476 & 280 & 0,967 & 0,639 \\
\hline 112 & 0,969 & 0,696 & 284 & 0,937 & 0,192 \\
\hline 116 & 0,984 & 0,968 & 288 & 0,943 & 0,233 \\
\hline 120 & 0,983 & 0,952 & 292 & 0,958 & 0,443 \\
\hline 124 & 0,939 & 0,189 & 296 & 0,951 & 0,355 \\
\hline 128 & 0,966 & 0,615 & 300 & 0,950 & 0,321 \\
\hline 132 & 0,978 & 0,874 & 304 & 0,957 & 0,440 \\
\hline 136 & 0,968 & 0,654 & 308 & 0,971 & 0,737 \\
\hline 140 & 0,987 & 0,987 & 312 & 0,985 & 0,974 \\
\hline 144 & 0,953 & 0,364 & 316 & 0,947 & 0,279 \\
\hline 148 & 0,945 & 0,246 & 320 & 0,948 & 0,294 \\
\hline 152 & 0,945 & 0,255 & 324 & 0,961 & 0,510 \\
\hline 156 & 0,946 & 0,258 & 328 & 0,962 & 0,528 \\
\hline 160 & 0,971 & 0,731 & 332 & 0,962 & 0,533 \\
\hline 164 & 0,979 & 0,898 & 336 & 0,967 & 0,638 \\
\hline 168 & 0,985 & 0,979 & 340 & 0,940 & 0,203 \\
\hline 172 & 0,953 & 0,380 & 344 & 0,983 & 0,958 \\
\hline 176 & 0,905 & 0,038 & 348 & 0,983 & 0,961 \\
\hline 180 & 0,966 & 0,628 & 352 & 0,967 & 0,648 \\
\hline 184 & 0,973 & 0,790 & 356 & 0,948 & 0,286 \\
\hline 188 & 0,985 & 0,976 & 360 & 0,966 & 0,613 \\
\hline 192 & 0,946 & 0,263 & & & \\
\hline
\end{tabular}


Figura 3.5. Perfil das médias, limites inferior e superior dos intervalos de confiança $(95 \%)$ da média e o perfil dos valores preditos para $\Delta \mathrm{VO}_{2}$ por um modelo monoexponencial ajustado.

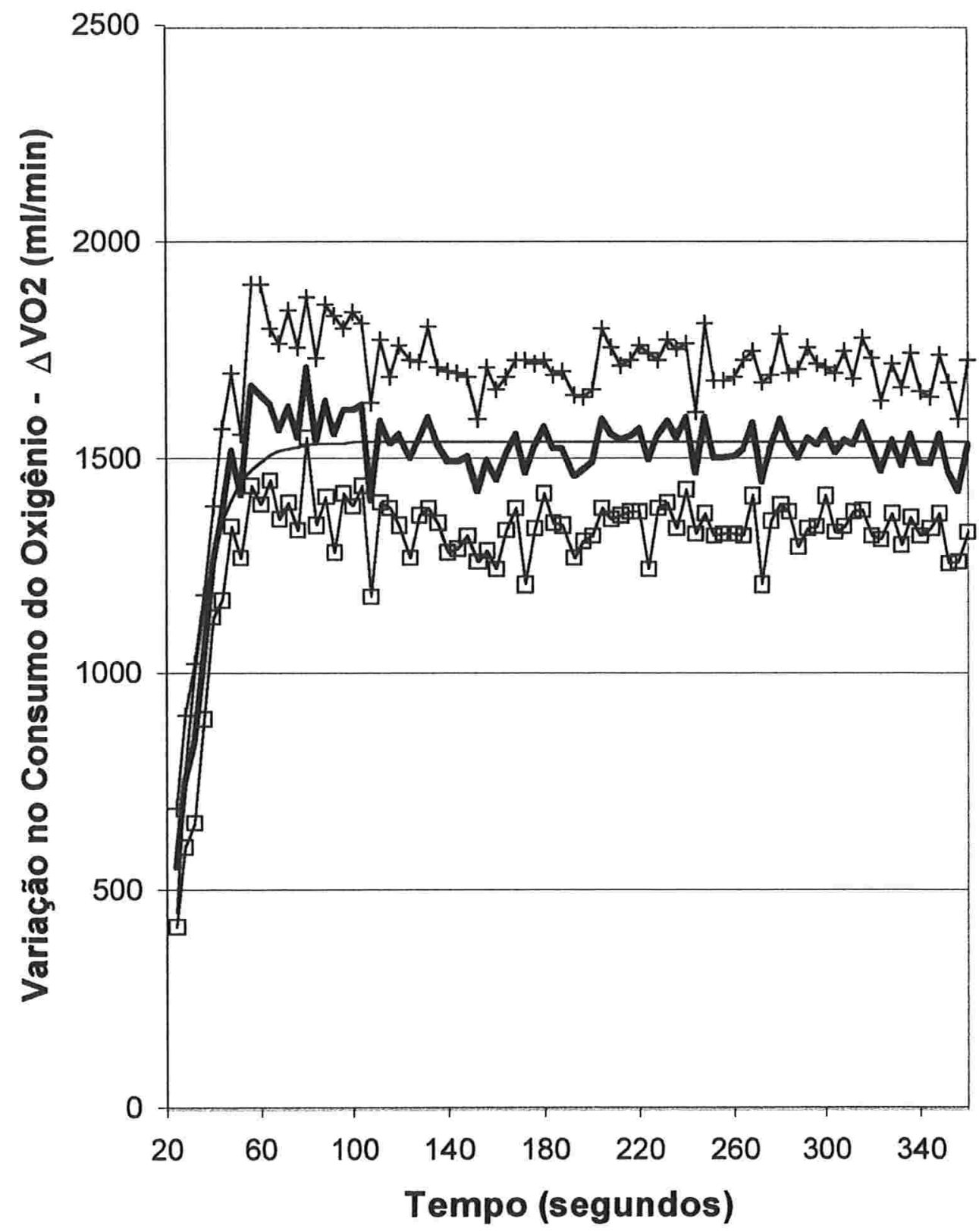

$\longrightarrow$ Média —Modelo $\square$ - Limite inferior $\longrightarrow$ Limite superior 
Figura 3.6. Valores da variação no consumo de oxigênio observados e respectivo perfil predito pelo modelo ajustado em cada atleta.

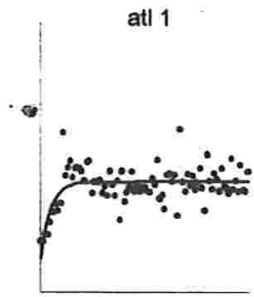

atl 7

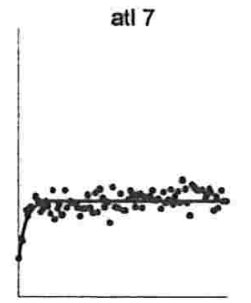

atl 13

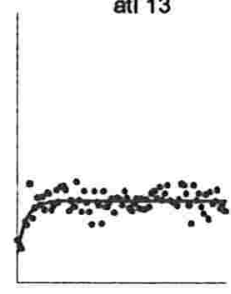

at! 20

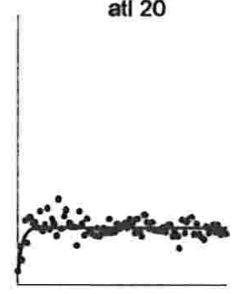

atl 26

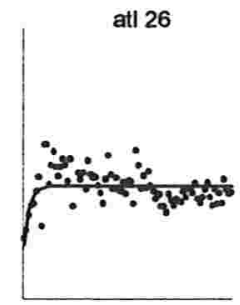

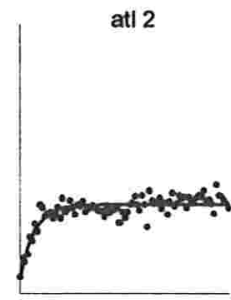

atl 9

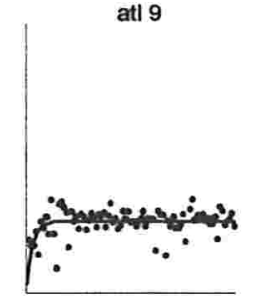

atl 14

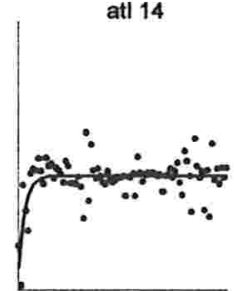

atl 22

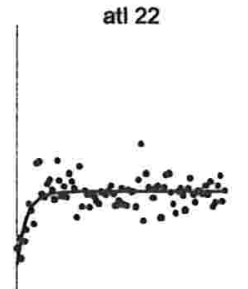

atl 29

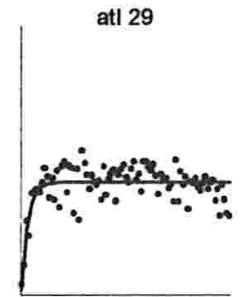

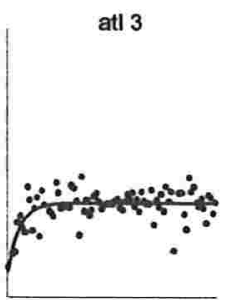

atl 10

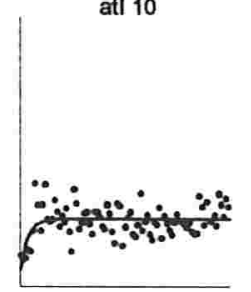

atl 15

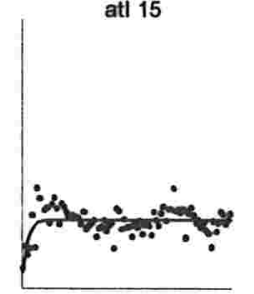

att 23

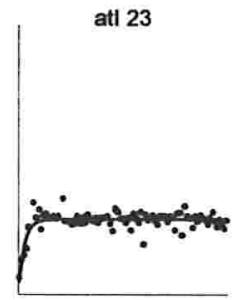

24

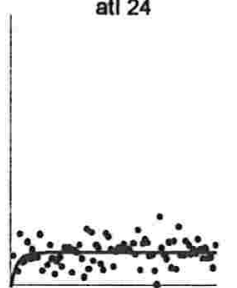

atl 6

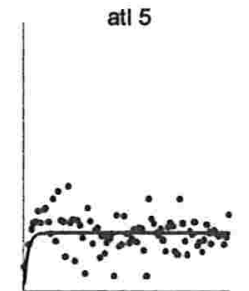

atl 11

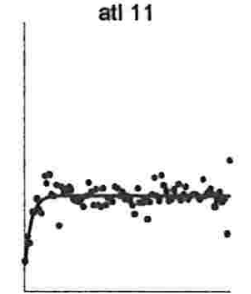

atl 17

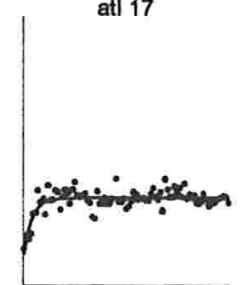

atl 19

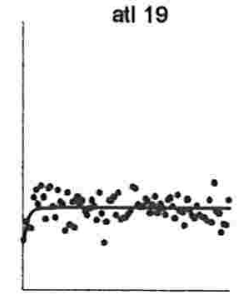

atl 25

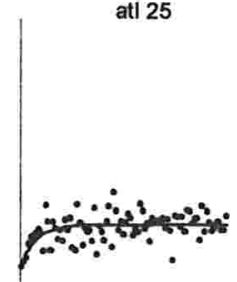

Eixo vertical: variação no consumo de oxigênio (0 a $5000 \mathrm{ml} / \mathrm{min}$ )

Eixo horizontal: tempo de corrida (24 a 360 segundos) 


\section{b. Ajuste do modelo}

Dentre as alternativas disponíveis, modelos da forma [1.1] com efeitos aleatórios foram escolhidos, dada a sua flexibilidade para modelar as curvas não lineares sob diversas estruturas de covariância (Davidian and Giltinan, 1998). Para a análise dos valores de $\Delta \mathrm{VO}_{2}$ de cada atleta, adotou-se o modelo [2.5] com

$$
a_{i}=\left(b_{i}, 0,0\right)^{t},
$$

que corresponde ao caso em que há apenas o efeito aleatório associado ao parâmetro $\beta$. Este modelo foi escolhido por ser de fácil implementação e não apresentar prejuízos à exemplificação da metodologia. $\mathrm{O}$ ajuste do modelo foi realizado com o auxílio do software estatístico SAS versão 8.02, PROC NLMIXED e envolveu as etapas descritas a seguir.

Na primeira etapa, estimaram-se os parâmetros $\left(\beta, \delta, \tau, \sigma_{\mathrm{b}}{ }^{2} \mathrm{e}\right.$ $\left.\sigma^{2}\right)$ por meio de máxima verossimilhança utilizando o método iterativo de Beal and Sheiner (1985). As informações obtidas durante o ajuste dos modelos mono-exponenciais [1.1] para as observações de cada atleta foram consideradas no processo de escolha dos valores iniciais necessários para o início do processo iterativo. As estimativas dos parâmetros, os respectivos erros padrão, os níveis descritivos e os correspondentes intervalos de confiança com coeficiente de confiança de $95 \%$ podem ser visualizados na Tabela 3.3. A matriz de covariância assintótica dos estimadores dos parâmetros está apresentada na Tabela 3.4. Na Figura 3.7 pode-se visualizar o ajuste deste modelo para os dados de cada indivíduo.

A análise dos preditores para os efeitos aleatórios $b_{i}$ baseou-se no teste de normalidade de Shapiro-Wilk. Não foram obtidos indícios 
Tabela 3.3. Estimativas dos parâmetros do modelo [2.5], erros padrão, valores p e respectivos limites dos intervalos de confiança com coeficiente de confiança de $95 \%$.

\begin{tabular}{cccccc}
\hline Parâmetro & Estimativa & Erro Padrão & Valor P & Limite Inf. & Limite Sup. \\
\hline$\beta$ & 1539,99 & 82,72 & $<0,001$ & 1367,97 & 1712,02 \\
$\delta$ & 19,83 & 0,59 & $<0,001$ & 18,61 & 21,06 \\
$\tau$ & 11,65 & 0,65 & $<0,001$ & 10,29 & 13,01 \\
$\sigma_{b}{ }^{2}$ & 149750 & 45327 & 0,003 & 55488 & 244012 \\
$\sigma^{2}$ & 57200 & 1889 & $<0,001$ & 53272 & 61128 \\
\hline
\end{tabular}

Tabela 3.4. Estimativa da matriz de covariância dos estimadores dos parâmetros do modelo [2.5].

\begin{tabular}{cccccc}
\hline parâmetros & $\beta$ & $\delta$ & $\tau$ & $\sigma_{b}^{2}$ & $\sigma^{2}$ \\
$\beta$ & 6842 & $-0,225$ & 0,831 & 45206 & $-0,056$ \\
$\delta$ & $-0,225$ & 0,347 & $-0,291$ & -59 & 0,054 \\
$\tau$ & 0,831 & $-0,291$ & 0,427 & 181 & $-0,061$ \\
$\sigma_{b}^{2}$ & 45206 & -59 & 181 & 2054536929 & -44194 \\
$\sigma^{2}$ & $-0,056$ & 0,054 & $-0,061$ & -44194 & 3566886 \\
\hline
\end{tabular}


Figura 3.7. Valores da variação no consumo de oxigênio mensurados e respectivo perfil predito pelo modelo [2.5] em cada atleta.

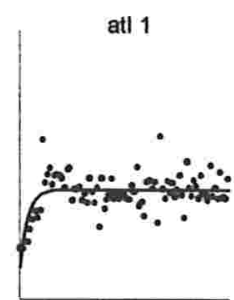

atl 7

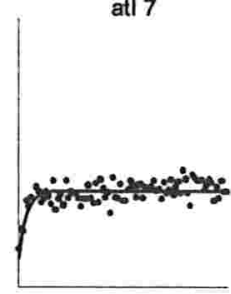

atl 13

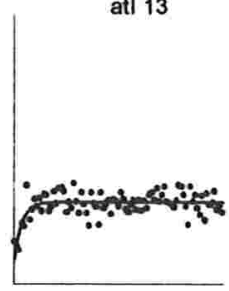

atl 20

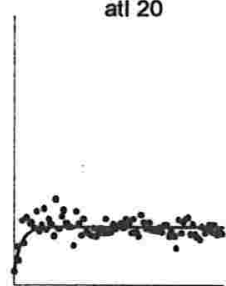

atl 26

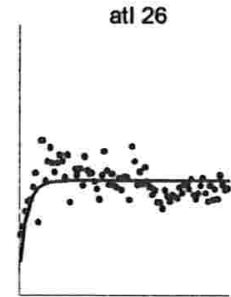

atl 2

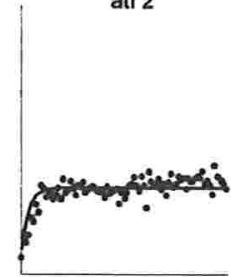

atl 9

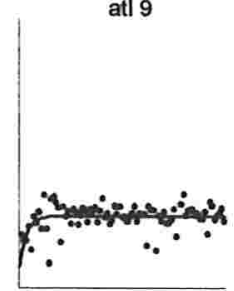

atl 14

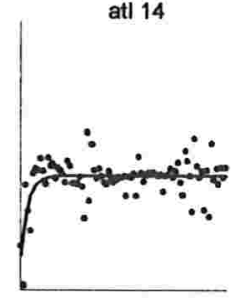

atl 22

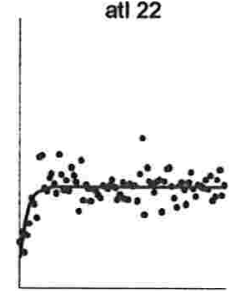

atl 29

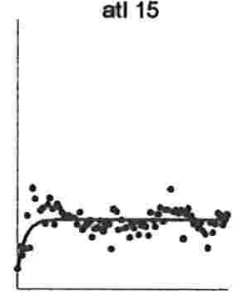

atl 23
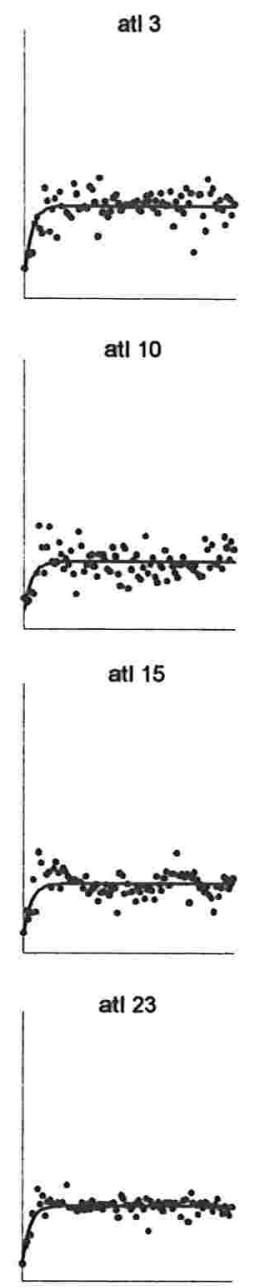

atl 10

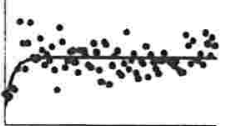

atl 15

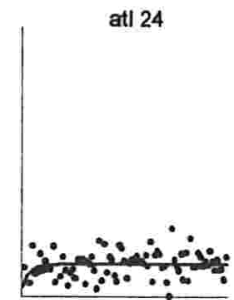

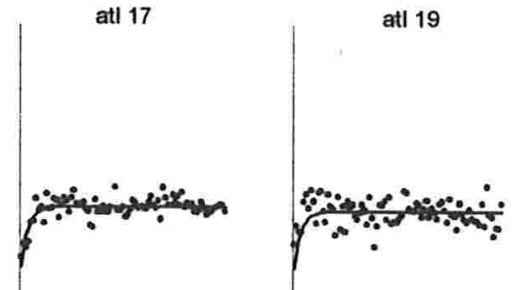

atl 24

atl 6

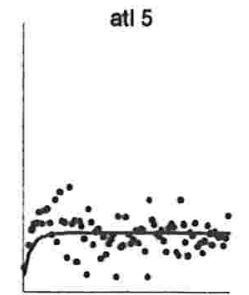

atl 11

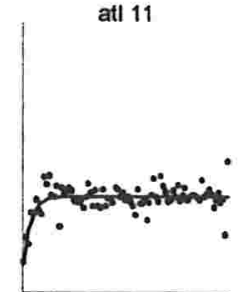

atl 17

atl 25

Eixo vertical: variação no consumo de oxigênio (0 a $5000 \mathrm{ml} / \mathrm{min})$ Eixo horizontal: tempo de corrida ( 24 a 360 segundos) 
contrários à adoção de um modelo probabilístico normal para a distribuição desses preditores $(\mathrm{p}=0,577)$. A análise dos resíduos também se baseou no teste de normalidade de Shapiro-Wilk e na análise do diagrama de dispersão dos resíduos ao longo do tempo (Figura 3.8), do histograma dos resíduos com curva normal sobreposta (Figura 3.9) e do gráfico normal quantil-quantil dos resíduos (Figura 3.10). Apesar de o teste de ShapiroWilk rejeitar a hipótese de que os resíduos obedecem a uma distribuição normal $(p<0,001)$, as demais análises não apontam nessa direção.

\section{c. Curvas de Referência}

Uma vez ajustado o modelo e obtidas as estimativas dos parâmetros em $\boldsymbol{\mu}=(\beta, \delta, \tau)^{\mathrm{t}}$ e $\mathbf{w}=\left(\sigma_{\mathrm{b}}^{2}, \sigma^{2}\right)^{\mathrm{t}}$, pode-se prosseguir com a construção das curvas de referência. Para a identificação de perfis extremos, a curva de referência de $100 \gamma \%(0<\gamma<1)$ é dada por [2.4] com os elementos de $\operatorname{DP}\left(\mathbf{Z}_{\mathrm{i}}(\boldsymbol{\mu}) \Delta \mathbf{Z}_{\mathrm{i}}(\boldsymbol{\mu})^{t}\right)$ dados por

$$
\sqrt{\left\{1-\exp \left[-\frac{(t-\delta)}{\tau}\right]\right\}^{2} \sigma_{b}^{2}}
$$

Substituindo os parâmetros em [2.4] por suas estimativas de máxima verossimilhança, a curva de referência para identificação de valores extremos pode ser obtida. Na Tabela 3.5 apresentam-se as estimativas de $\mathbf{f}\left(\mathbf{t}_{\mathrm{i}}, \boldsymbol{\mu}, \boldsymbol{0}\right)$ e de $\operatorname{DP}\left(\mathbf{Z}_{\mathrm{i}}(\boldsymbol{\mu}) \Delta \mathbf{Z}_{\mathrm{i}}(\boldsymbol{\mu})^{\dagger}\right)$, assim como as estimativas para a matriz de covariâncias dos estimadores de $\mathbf{f}\left(\mathbf{t}_{\mathrm{i}}, \boldsymbol{\mu}, \mathbf{0}\right)$ e $\operatorname{DP}\left(\mathbf{Z}_{\mathrm{i}}(\boldsymbol{\mu}) \Delta \mathbf{Z}_{\mathrm{i}}(\boldsymbol{\mu})^{\dagger}\right)$. Estas estimativas foram calculadas computacionalmente por meio do método delta (Sen and Singer, 1993). 
Figura 3.8. Resíduos do modelo [2.5] para a variação no consumo de oxigênio ao longo do tempo.

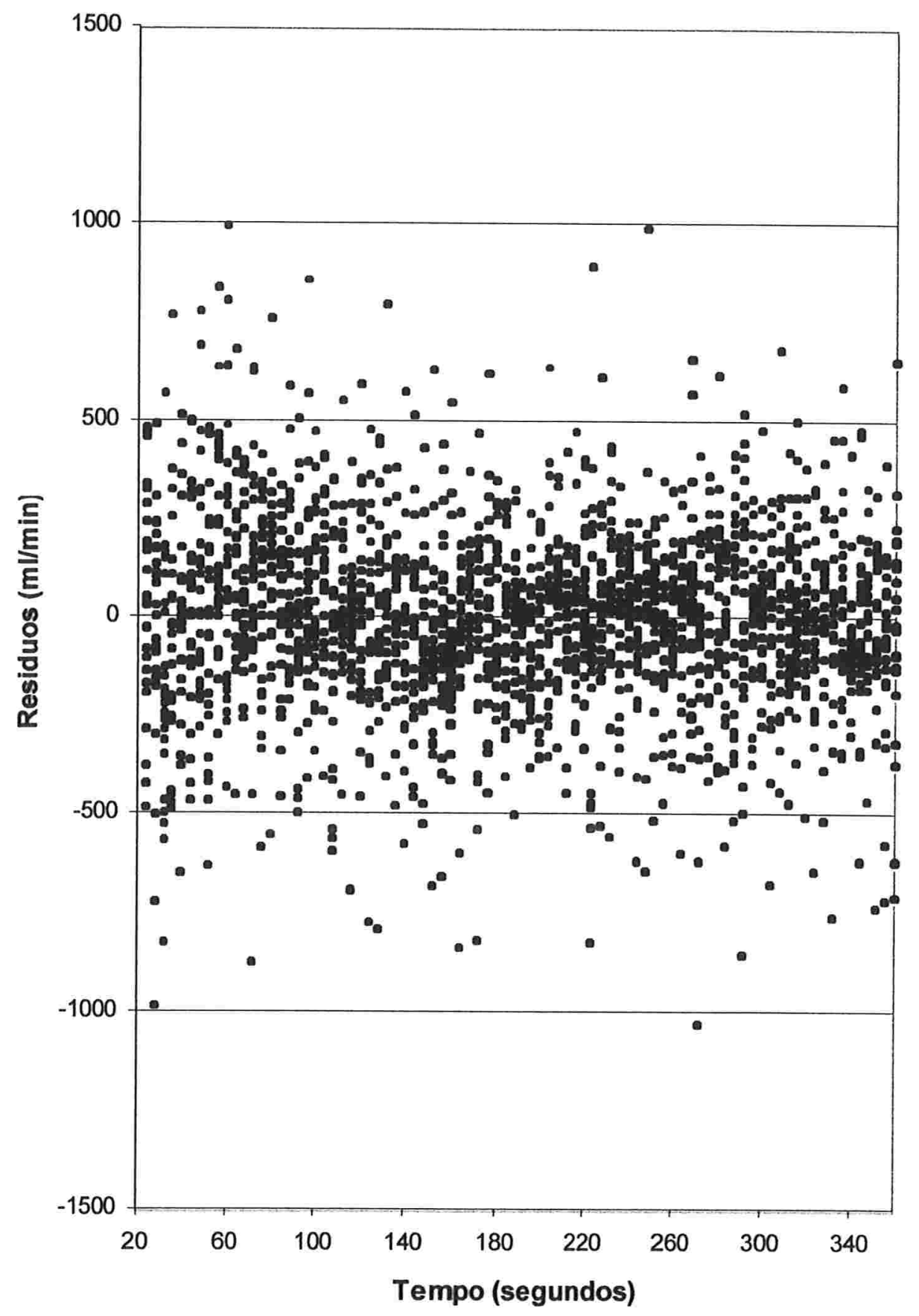


Figura 3.9. Histograma dos resíduos do modelo [2.5] para a variação no consumo de oxigênio sobreposto a uma curva normal.

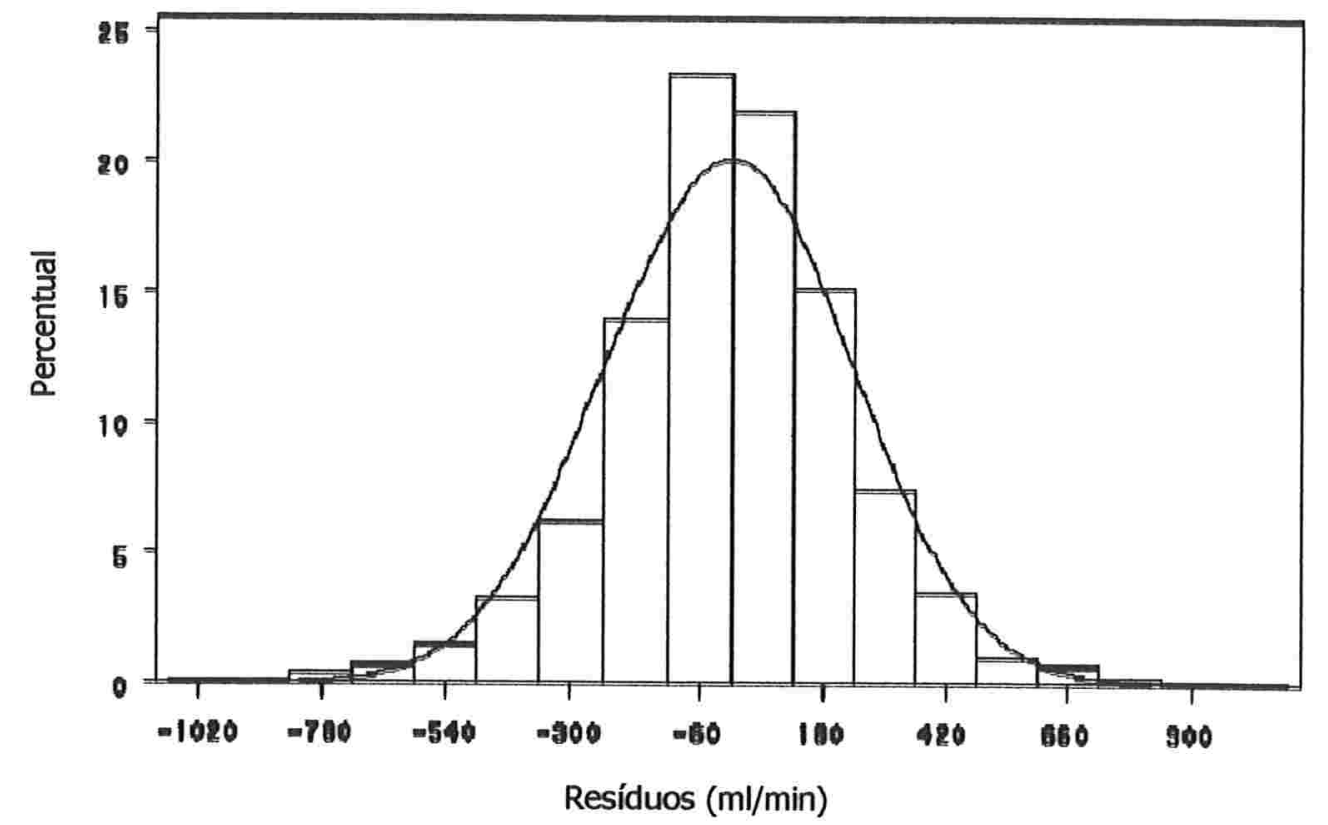

Figura 3.10. Gráfico Normal Q-Q dos resíduos do modelo [2.5] para a variação no consumo de oxigênio.

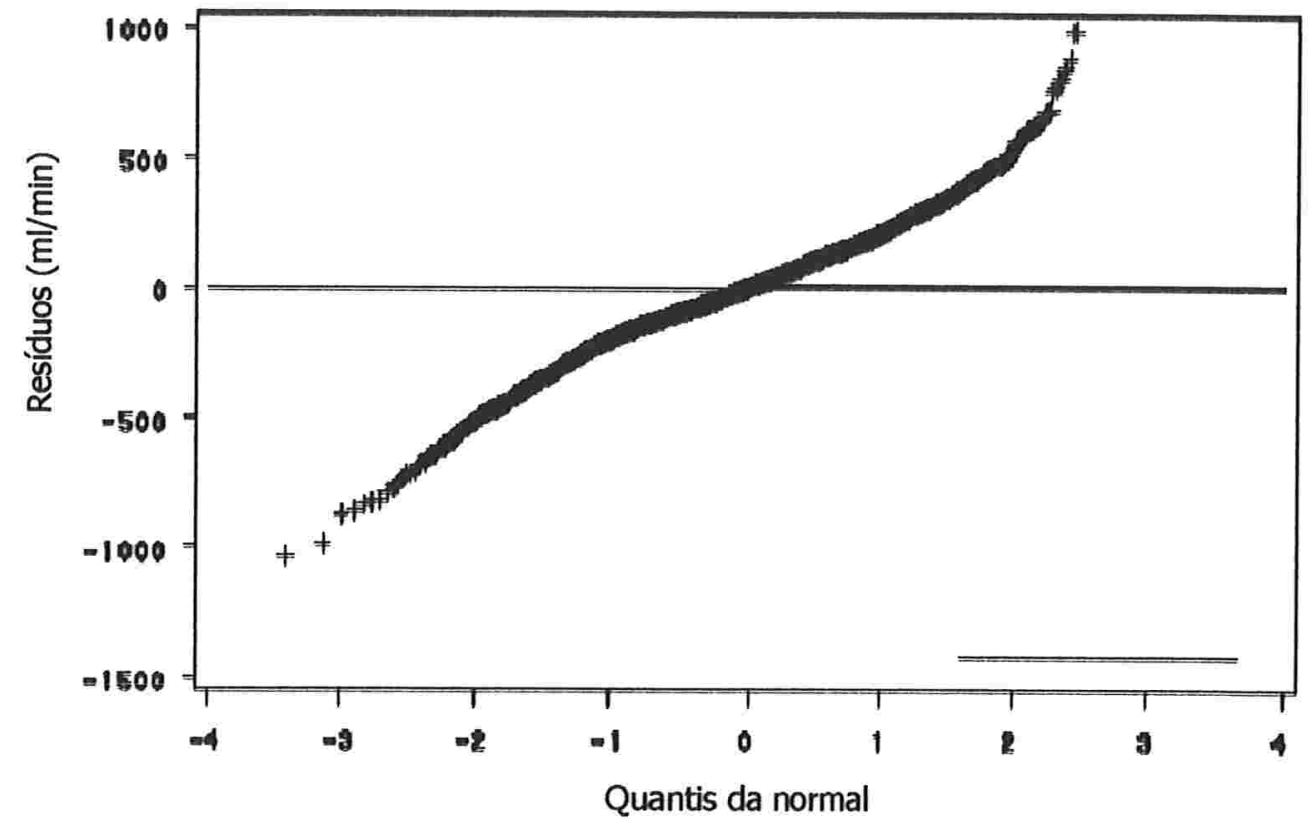


Tabela 3.5. Estimativas de $\mathbf{f}\left(\mathbf{t}_{\mathrm{i}}, \boldsymbol{\mu}, \mathbf{0}\right), \operatorname{DP}\left(\mathbf{Z}_{\mathrm{i}}(\boldsymbol{\mu}) \Delta \mathbf{Z}_{\mathrm{i}}(\boldsymbol{\mu})^{t}\right)$ e de suas variâncias e covariância obtidas sob o modelo [2.5], em cada momento.

\begin{tabular}{|c|c|c|c|c|c|}
\hline Tempo & $f(\mathbf{4}, \mu, 0)$ & $\operatorname{DP}\left(Z_{i}(\mu) \cdot \Delta \cdot Z_{i}(\mu)^{t}\right)$ & $\operatorname{Var}\left\{f\left(t_{u}, \mu, 0\right)\right\}$ & $\operatorname{Var}\left\{D P\left(Z_{i}(\mu) \cdot \Delta \cdot Z_{i}(\mu)^{t}\right)\right\}$ & $\operatorname{Cov}\left\{f\left(t_{i} \mu, 0\right), D P\left(Z_{i}(\mu) \cdot \Delta \cdot Z_{i}(\mu)^{t}\right)\right\}$ \\
\hline 24 & 463 & 212 & 2269 & 1118 & 388 \\
\hline 28 & 776 & 275 & 2354 & 1747 & 128 \\
\hline 32 & 998 & 312 & 3278 & 2232 & 90 \\
\hline 36 & 1156 & 335 & 4200 & 2580 & 85 \\
\hline 40 & 1267 & 351 & 4926 & 2827 & 80 \\
\hline 44 & 1347 & 362 & 5457 & 3002 & 75 \\
\hline 48 & 1403 & 369 & 5838 & 3126 & 69 \\
\hline 52 & 1443 & 375 & 6112 & 3214 & 64 \\
\hline 56 & 1471 & 378 & 6309 & 3277 & 61 \\
\hline 60 & 1491 & 381 & 6453 & 3321 & 59 \\
\hline 64 & 1505 & 383 & 6558 & 3353 & 58 \\
\hline 68 & 1515 & 384 & 6635 & 3375 & 57 \\
\hline 72 & 1523 & 385 & 6692 & 3391 & 57 \\
\hline 76 & 1528 & 385 & 6733 & 3402 & 57 \\
\hline 80 & 1531 & 386 & 6763 & 3410 & 57 \\
\hline 84 & 1534 & 386 & 6785 & 3416 & 57 \\
\hline 88 & 1536 & 386 & 6801 & 3420 & 58 \\
\hline 92 & 1537 & 387 & 6813 & 3423 & 58 \\
\hline 96 & 1538 & 387 & 6821 & 3425 & 58 \\
\hline 100 & 1538 & 387 & 6827 & 3426 & 58 \\
\hline 104 & 1539 & 387 & 6832 & 3427 & 58 \\
\hline 108 & 1539 & 387 & 6835 & 3428 & 58 \\
\hline 112 & 1539 & 387 & 6837 & 3429 & 58 \\
\hline 116 & 1540 & 387 & 6838 & 3429 & 58 \\
\hline 120 & 1540 & 387 & 6840 & 3429 & 58 \\
\hline 124 & 1540 & 387 & 6840 & 3429 & 58 \\
\hline 128 & 1540 & 387 & 6841 & 3430 & 58 \\
\hline 132 & 1540 & 387 & 6841 & 3430 & 58 \\
\hline 136 & 1540 & 387 & 6842 & 3430 & 58 \\
\hline 140 & 1540 & 387 & 6842 & 3430 & 58 \\
\hline 144 & 1540 & 387 & 6842 & 3430 & 58 \\
\hline 148 & 1540 & 387 & 6842 & 3430 & 58 \\
\hline 152 & 1540 & 387 & 6842 & 3430 & 58 \\
\hline 156 & 1540 & 387 & 6842 & 3430 & 58 \\
\hline 160 & 1540 & 387 & 6842 & 3430 & 58 \\
\hline 164 & 1540 & 387 & 6842 & 3430 & 58 \\
\hline 168 & 1540 & 387 & 6842 & 3430 & 58 \\
\hline 172 & 1540 & 387 & 6842 & 3430 & 58 \\
\hline 176 & 1540 & 387 & 6842 & 3430 & 58 \\
\hline 180 & 1540 & 387 & 6842 & 3430 & 58 \\
\hline 184 & 1540 & 387 & 6842 & 3430 & 58 \\
\hline 188 & 1540 & 387 & 6842 & 3430 & 58 \\
\hline 192 & 1540 & 387 & 6842 & 3430 & 58 \\
\hline 196 & 1540 & 387 & 6842 & 3430 & 58 \\
\hline 200 & 1540 & 387 & 6842 & 3430 & 58 \\
\hline 204 & 1540 & 387 & 6842 & 3430 & 58 \\
\hline 208 & 1540 & 387 & 6842 & 3430 & 58 \\
\hline 212 & 1540 & 387 & 6842 & 3430 & 58 \\
\hline 216 & 1540 & 387 & 6842 & 3430 & 58 \\
\hline 220 & 1540 & 387 & 6842 & 3430 & 58 \\
\hline 224 & 1540 & 387 & 6842 & 3430 & 58 \\
\hline 228 & 1540 & 387 & 6842 & 3430 & 58 \\
\hline
\end{tabular}


Tabela 3.5. (Cont.) Estimativas de $\mathbf{f}\left(\mathbf{t}_{\mathrm{i}}, \boldsymbol{\mu}, \mathbf{0}\right), \operatorname{DP}\left(\mathbf{Z}_{\mathrm{i}}(\boldsymbol{\mu}) \Delta \mathbf{Z}_{\mathrm{i}}(\boldsymbol{\mu})^{t}\right)$ e de suas variâncias e covariância obtidas sob o modelo [2.5], em cada momento.

\begin{tabular}{|c|c|c|c|c|c|}
\hline Tempo & $f(\boldsymbol{t}, \mu, 0)$ & $\operatorname{DP}\left(Z_{i}(\mu) \cdot \Delta \cdot Z_{i}(\mu)\right)$ & $\operatorname{Var}\{f(t, \mu, 0)\}$ & $\operatorname{Var}\left\{\operatorname{DP}\left(Z_{i}(\mu) \cdot \Delta \cdot Z_{i}(\mu)^{t}\right)\right\}$ & $\operatorname{Cov}\left\{f\left(t_{1}, \mu, 0\right), \operatorname{DP}\left(Z_{i}(\mu) \cdot \Delta \cdot Z_{i}(\mu)^{t}\right)\right\}$ \\
\hline 232 & 1540 & 387 & 6842 & 3430 & 58 \\
\hline 236 & 1540 & 387 & 6842 & 3430 & 58 \\
\hline 240 & 1540 & 387 & 6842 & 3430 & 58 \\
\hline 244 & 1540 & 387 & 6842 & 3430 & 58 \\
\hline 248 & 1540 & 387 & 6842 & 3430 & 58 \\
\hline 252 & 1540 & 387 & 6842 & 3430 & 58 \\
\hline 256 & 1540 & 387 & 6842 & 3430 & 58 \\
\hline 260 & 1540 & 387 & 6842 & 3430 & 58 \\
\hline 264 & 1540 & 387 & 6842 & 3430 & 58 \\
\hline 268 & 1540 & 387 & 6842 & 3430 & 58 \\
\hline 272 & 1540 & 387 & 6842 & 3430 & 58 \\
\hline 276 & 1540 & 387 & 6842 & 3430 & 58 \\
\hline 280 & 1540 & 387 & 6842 & 3430 & 58 \\
\hline 284 & 1540 & 387 & 6842 & 3430 & 58 \\
\hline 288 & 1540 & 387 & 6842 & 3430 & 58 \\
\hline 292 & 1540 & 387 & 6842 & 3430 & 58 \\
\hline 296 & 1540 & 387 & 6842 & 3430 & 58 \\
\hline 300 & 1540 & 387 & 6842 & 3430 & 58 \\
\hline 304 & 1540 & 387 & 6842 & 3430 & 58 \\
\hline 308 & 1540 & 387 & 6842 & 3430 & 58 \\
\hline 312 & 1540 & 387 & 6842 & 3430 & 58 \\
\hline 316 & 1540 & 387 & 6842 & 3430 & 58 \\
\hline 320 & 1540 & 387 & 6842 & 3430 & 58 \\
\hline 324 & 1540 & 387 & 6842 & 3430 & 58 \\
\hline 328 & 1540 & 387 & 6842 & 3430 & 58 \\
\hline 332 & 1540 & 387 & 6842 & 3430 & 58 \\
\hline 336 & 1540 & 387 & 6842 & 3430 & 58 \\
\hline 340 & 1540 & 387 & 6842 & 3430 & 58 \\
\hline 344 & 1540 & 387 & 6842 & 3430 & 58 \\
\hline 348 & 1540 & 387 & 6842 & 3430 & 58 \\
\hline 352 & 1540 & 387 & 6842 & 3430 & 58 \\
\hline 356 & 1540 & 387 & 6842 & 3430 & 58 \\
\hline 360 & 1540 & 387 & 6842 & 3430 & 58 \\
\hline
\end{tabular}


Substituindo as estimativas de $\mathbf{f}\left(\mathbf{t}_{\mathrm{i}}, \boldsymbol{\mu}, \mathbf{0}\right)$ e $\operatorname{DP}\left(\mathbf{Z}_{\mathrm{i}}(\boldsymbol{\mu}) \Delta \mathbf{Z}_{\mathrm{i}}(\boldsymbol{\mu})^{t}\right)$ em [2.4] para cada momento de observação, calculam-se as respectivas curvas de referência de $10 \%, 25 \%, 50 \%, 75 \%$ e $90 \%$. Essas curvas de referência estão relacionadas na Tabela 3.6 e podem ser visualizadas na Figura 3.11.

Com o objetivo de avaliar o ajuste das estimativas das curvas de referência, todos os indivíduos foram comparados com as curvas de referência de $10 \%, 25 \%, 50 \%, 75 \%$ e 90\%. A Figura 3.12, apresenta a alguns perfis individuais e as curvas de referência estimadas. Na Tabela 3.7 apresentamos as quantidades de clientes considerados abaixo das curvas de referência por dois critérios: (i) ou se mais de $50 \%$ de suas observações apresentam valores inferiores aos da curva de referência, (ii) ou se mais de $50 \%$ dos valores preditos sob o modelo [1.1] ajustado por meio de mínimos quadrados ordinários, são inferiores.

Intervalos de confiança com coeficiente de confiança de $95 \%$ também foram calculados para as curvas de referência de $10 \%, 50 \%$ e $90 \%$. $\mathrm{O}$ cálculo desses intervalos de confiança foi realizado pelo procedimento descrito no capítulo anterior. As estimativas para os valores de $\operatorname{DP}\left(\operatorname{Var}\left[\widehat{\mathbf{y}_{\gamma}}\right]\right)$ foram calculadas utilizando os valores da Tabela 3.5 e são apresentados na Tabela 3.8. Na Figura 3.13, as curvas de referência de $10 \%, 50 \%$ e $90 \%$ e seus respectivos intervalos de confiança podem ser visualizados.

Na Tabela 3.7, podemos observar que a maior diferença entre os percentuais de indivíduos abaixo das curvas de referência e o percentil esperado com a curva de referência ocorre para o percentil $90 \%$. Porém, esse ajuste parece melhorar para os demais percentis. 
Na Figura 3.13, observamos entre os intervalos de confiança calculados tem uma amplitude em torno de $400 \mathrm{ml} / \mathrm{min}$ que pode indicar uma amostra de tamanho insuficiente para uma estimação acurada das curvas de referência, contudo suficiente para a demonstração desta metodologia. 
Tabela 3.6. Curvas de referência aproximadas de $10 \%, 25 \%, 50 \%, 75 \%$ e $90 \%$ para a variação no consumo de oxigênio, obtidas sob o modelo [2.5] e segundo a equação [2.4], em cada momento.

\begin{tabular}{|c|c|c|c|c|c|}
\hline Tempo & $\mathbf{v}_{10 \%}$ & $\mathbf{y}_{25 \%}$ & $\mathbf{v}_{50 \%}$ & $\mathbf{y}_{75 \%}$ & $\mathbf{V}_{90 \%}$ \\
\hline 24 & 191 & 320 & 463 & 606 & 735 \\
\hline 28 & 424 & 591 & 776 & 961 & 1128 \\
\hline 32 & 599 & 788 & 998 & 1208 & 1397 \\
\hline 36 & 726 & 930 & 1156 & 1382 & 1585 \\
\hline 40 & 817 & 1031 & 1267 & 1504 & 1717 \\
\hline 44 & 883 & 1102 & 1347 & 1591 & 1810 \\
\hline 48 & 929 & 1154 & 1403 & 1652 & 1876 \\
\hline 52 & 963 & 1190 & 1443 & 1695 & 1923 \\
\hline 56 & 986 & 1216 & 1471 & 1726 & 1956 \\
\hline 60 & 1003 & 1234 & 1491 & 1748 & 1979 \\
\hline 64 & 1015 & 1247 & 1505 & 1763 & 1996 \\
\hline 68 & 1023 & 1256 & 1515 & 1774 & 2007 \\
\hline 72 & 1029 & 1263 & 1523 & 1782 & 2016 \\
\hline 76 & 1034 & 1268 & 1528 & 1788 & 2022 \\
\hline 80 & 1037 & 1271 & 1531 & 1791 & 2026 \\
\hline 84 & 1039 & 1273 & 1534 & 1794 & 2029 \\
\hline 88 & 1040 & 1275 & 1536 & 1796 & 2031 \\
\hline 92 & 1041 & 1276 & 1537 & 1798 & 2032 \\
\hline 96 & 1042 & 1277 & 1538 & 1799 & 2033 \\
\hline 100 & 1043 & 1278 & 1538 & 1799 & 2034 \\
\hline 104 & 1043 & 1278 & 1539 & 1800 & 2035 \\
\hline 108 & 1043 & 1278 & 1539 & 1800 & 2035 \\
\hline 112 & 1044 & 1278 & 1539 & 1800 & 2035 \\
\hline 116 & 1044 & 1279 & 1540 & 1801 & 2035 \\
\hline 120 & 1044 & 1279 & 1540 & 1801 & 2036 \\
\hline 124 & 1044 & 1279 & 1540 & 1801 & 2036 \\
\hline 128 & 1044 & 1279 & 1540 & 1801 & 2036 \\
\hline 132 & 1044 & 1279 & 1540 & 1801 & 2036 \\
\hline 136 & 1044 & 1279 & 1540 & 1801 & 2036 \\
\hline 140 & 1044 & 1279 & 1540 & 1801 & 2036 \\
\hline 144 & 1044 & 1279 & 1540 & 1801 & 2036 \\
\hline 148 & 1044 & 1279 & 1540 & 1801 & 2036 \\
\hline 152 & 1044 & 1279 & 1540 & 1801 & 2036 \\
\hline 156 & 1044 & 1279 & 1540 & 1801 & 2036 \\
\hline 160 & 1044 & 1279 & 1540 & 1801 & 2036 \\
\hline 164 & 1044 & 1279 & 1540 & 1801 & 2036 \\
\hline 168 & 1044 & 1279 & 1540 & 1801 & 2036 \\
\hline 172 & 1044 & 1279 & 1540 & 1801 & 2036 \\
\hline 176 & 1044 & 1279 & 1540 & 1801 & 2036 \\
\hline 180 & 1044 & 1279 & 1540 & 1801 & 2036 \\
\hline 184 & 1044 & 1279 & 1540 & 1801 & 2036 \\
\hline 188 & 1044 & 1279 & 1540 & 1801 & 2036 \\
\hline 192 & 1044 & 1279 & 1540 & 1801 & 2036 \\
\hline 196 & 1044 & 1279 & 1540 & 1801 & 2036 \\
\hline 200 & 1044 & 1279 & 1540 & 1801 & 2036 \\
\hline 204 & 1044 & 1279 & 1540 & 1801 & 2036 \\
\hline 208 & 1044 & 1279 & 1540 & 1801 & 2036 \\
\hline 212 & 1044 & 1279 & 1540 & 1801 & 2036 \\
\hline 216 & 1044 & 1279 & 1540 & 1801 & 2036 \\
\hline 220 & 1044 & 1279 & 1540 & 1801 & 2036 \\
\hline 224 & 1044 & 1279 & 1540 & 1801 & 2036 \\
\hline 228 & 1044 & 1279 & 1540 & 1801 & 2036 \\
\hline
\end{tabular}


Tabela 3.6. (Cont.) Curvas de referência aproximadas de 10\%, 25\%, 50\%, $75 \%$ e $90 \%$ para a variação no consumo de oxigênio, obtidas sob o modelo [2.5] e segundo a equação [2.4], em cada momento.

\begin{tabular}{|c|c|c|c|c|c|}
\hline Tempo & $\mathbf{y}_{10 \%}$ & $\mathbf{y}_{25 \%}$ & $Y_{50 \%}$ & $Y_{75 \%}$ & $Y_{90 \%}$ \\
\hline 232 & 1044 & 1279 & 1540 & 1801 & 2036 \\
\hline 236 & 1044 & 1279 & 1540 & 1801 & 2036 \\
\hline 240 & 1044 & 1279 & 1540 & 1801 & 2036 \\
\hline 244 & 1044 & 1279 & 1540 & 1801 & 2036 \\
\hline 248 & 1044 & 1279 & 1540 & 1801 & 2036 \\
\hline 252 & 1044 & 1279 & 1540 & 1801 & 2036 \\
\hline 256 & 1044 & 1279 & 1540 & 1801 & 2036 \\
\hline 260 & 1044 & 1279 & 1540 & 1801 & 2036 \\
\hline 264 & 1044 & 1279 & 1540 & 1801 & 2036 \\
\hline 268 & 1044 & 1279 & 1540 & 1801 & 2036 \\
\hline 272 & 1044 & 1279 & 1540 & 1801 & 2036 \\
\hline 276 & 1044 & 1279 & 1540 & 1801 & 2036 \\
\hline 280 & 1044 & 1279 & 1540 & 1801 & 2036 \\
\hline 284 & 1044 & 1279 & 1540 & 1801 & 2036 \\
\hline 288 & 1044 & 1279 & 1540 & 1801 & 2036 \\
\hline 292 & 1044 & 1279 & 1540 & 1801 & 2036 \\
\hline 296 & 1044 & 1279 & 1540 & 1801 & 2036 \\
\hline 300 & 1044 & 1279 & 1540 & 1801 & 2036 \\
\hline 304 & 1044 & 1279 & 1540 & 1801 & 2036 \\
\hline 308 & 1044 & 1279 & 1540 & 1801 & 2036 \\
\hline 312 & 1044 & 1279 & 1540 & 1801 & 2036 \\
\hline 316 & 1044 & 1279 & 1540 & 1801 & 2036 \\
\hline 320 & 1044 & 1279 & 1540 & 1801 & 2036 \\
\hline 324 & 1044 & 1279 & 1540 & 1801 & 2036 \\
\hline 328 & 1044 & 1279 & 1540 & 1801 & 2036 \\
\hline 332 & 1044 & 1279 & 1540 & 1801 & 2036 \\
\hline 336 & 1044 & 1279 & 1540 & 1801 & 2036 \\
\hline 340 & 1044 & 1279 & 1540 & 1801 & 2036 \\
\hline 344 & 1044 & 1279 & 1540 & 1801 & 2036 \\
\hline 348 & 1044 & 1279 & 1540 & 1801 & 2036 \\
\hline 352 & 1044 & 1279 & 1540 & 1801 & 2036 \\
\hline 356 & 1044 & 1279 & 1540 & 1801 & 2036 \\
\hline 360 & 1044 & 1279 & 1540 & 1801 & 2036 \\
\hline
\end{tabular}


Figura 3.11. Curvas de referência aproximadas de $10 \%, 25 \%, 50 \%, 75 \%$ e $90 \%$ para a variação no consumo de oxigênio, obtidas sob o modelo [2.5] e segundo a equação [2.4].

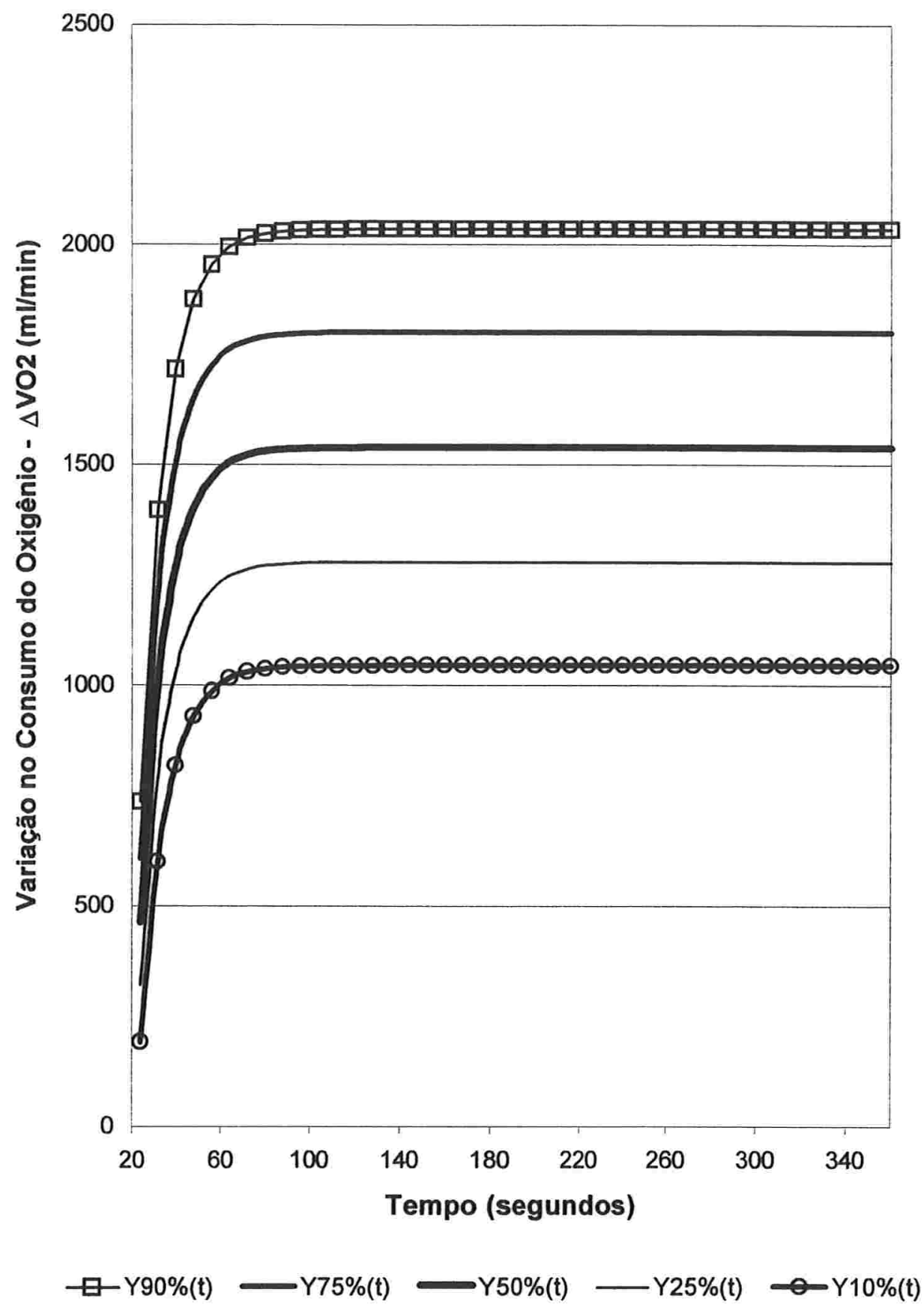


Figura 3.12. Amostra de perfis individuais da variação no consumo de oxigênio $(\mathrm{ml} / \mathrm{min})$ sobrepostos às curvas de referência aproximadas de $10 \%, 25 \%, 50 \%$, $75 \%$ e $90 \%$ nos primeiros 180 segundos.

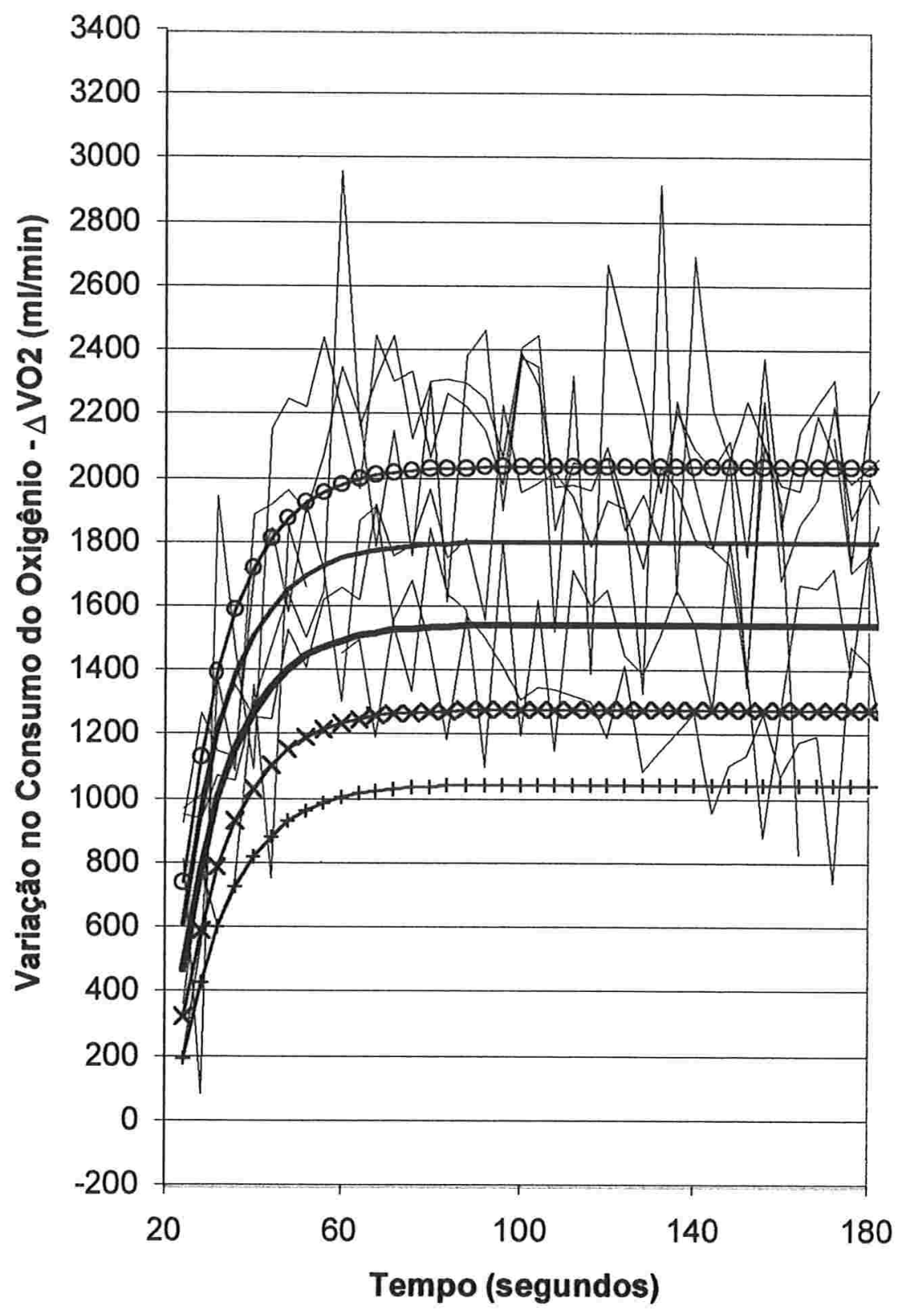

$-\mathrm{O} Y 90 \%(\mathrm{t})-\mathrm{Y} 75 \%(\mathrm{t})-Y 50 \%(\mathrm{t})-\mathrm{x}-\mathrm{Y} 25 \%(\mathrm{t}) \longrightarrow \mathrm{Y} 10 \%(\mathrm{t})$ 
Tabela 3.7. Comparação entre os indivíduos e as curvas de referência para os percentis de $10 \%, 25 \%, 50 \%, 75 \%$ e $90 \%$ por dois critérios: valores observados em cada indivíduo e valores preditos sob o modelo [1.1] ajustado para cada indivíduo por meio de mínimos quadrados ordinários.

\begin{tabular}{ccccc}
\hline & \multicolumn{2}{c}{ Valor Observado } & \multicolumn{2}{c}{ Valor Predito } \\
$\gamma \%$ & $\mathbf{N}$ & $\%$ & $\mathbf{N}$ & $\%$ \\
\hline 90 & 17 & 77 & 17 & 77 \\
75 & 16 & 73 & 16 & 73 \\
50 & 10 & 45 & 11 & 50 \\
25 & 7 & 32 & 7 & 32 \\
10 & 2 & 9 & 1 & 5 \\
\hline
\end{tabular}


Tabela 3.8. Limites superior e inferior para os intervalos de confiança com coeficiente de confiança de $95 \%$ para pontos escolhidos nas curvas de referência aproximadas de $10 \%, 50 \%$ e $90 \%$.

\begin{tabular}{|c|c|c|c|c|c|c|}
\hline \multirow[b]{2}{*}{ Tempo } & \multicolumn{2}{|c|}{$Y_{10 \%}$} & \multicolumn{2}{|c|}{$\mathrm{Y}_{50 \%}$} & \multicolumn{2}{|c|}{$Y_{90 \%}$} \\
\hline & L. inferior & L. superior & L.inferior & L. superior & L.inferior & L. superior \\
\hline 24 & 82 & 300 & 370 & 556 & 595 & 875 \\
\hline 28 & 287 & 561 & 681 & 871 & 982 & 1274 \\
\hline 32 & 438 & 759 & 886 & 1110 & 1231 & 1563 \\
\hline 36 & 548 & 904 & 1029 & 1283 & 1403 & 1768 \\
\hline 40 & 628 & 1007 & 1130 & 1405 & 1523 & 1911 \\
\hline 44 & 685 & 1081 & 1202 & 1491 & 1609 & 2012 \\
\hline 48 & 726 & 1133 & 1253 & 1553 & 1669 & 2083 \\
\hline 52 & 755 & 1170 & 1289 & 1596 & 1712 & 2133 \\
\hline 56 & 776 & 1197 & 1315 & 1627 & 1742 & 2169 \\
\hline 60 & 791 & 1216 & 1334 & 1648 & 1764 & 2194 \\
\hline 64 & 801 & 1229 & 1347 & 1664 & 1779 & 2212 \\
\hline 68 & 808 & 1238 & 1356 & 1675 & 1790 & 2225 \\
\hline 72 & 814 & 1245 & 1362 & 1683 & 1797 & 2234 \\
\hline 76 & 817 & 1250 & 1367 & 1688 & 1803 & 2240 \\
\hline 80 & 820 & 1253 & 1370 & 1692 & 1806 & 2245 \\
\hline 84 & 822 & 1256 & 1372 & 1695 & 1809 & 2248 \\
\hline 88 & 823 & 1257 & 1374 & 1697 & 1811 & 2250 \\
\hline 92 & 824 & 1259 & 1375 & 1699 & 1812 & 2252 \\
\hline 96 & 825 & 1260 & 1376 & 1700 & 1813 & 2253 \\
\hline 100 & 825 & 1260 & 1376 & 1700 & 1814 & 2254 \\
\hline 104 & 826 & 1261 & 1377 & 1701 & 1815 & 2255 \\
\hline 108 & 826 & 1261 & 1377 & 1701 & 1815 & 2255 \\
\hline 112 & 826 & 1261 & 1377 & 1701 & 1815 & 2255 \\
\hline 116 & 826 & 1261 & 1378 & 1702 & 1815 & 2256 \\
\hline 120 & 826 & 1261 & 1378 & 1702 & 1815 & 2256 \\
\hline 124 & 826 & 1261 & 1378 & 1702 & 1815 & 2256 \\
\hline 128 & 826 & 1262 & 1378 & 1702 & 1816 & 2256 \\
\hline 132 & 826 & 1262 & 1378 & 1702 & 1816 & 2256 \\
\hline 136 & 826 & 1262 & 1378 & 1702 & 1816 & 2256 \\
\hline 140 & 826 & 1262 & 1378 & 1702 & 1816 & 2256 \\
\hline 144 & 826 & 1262 & 1378 & 1702 & 1816 & 2256 \\
\hline 148 & 826 & 1262 & 1378 & 1702 & 1816 & 2256 \\
\hline 152 & 826 & 1262 & 1378 & 1702 & 1816 & 2256 \\
\hline 156 & 826 & 1262 & 1378 & 1702 & 1816 & 2256 \\
\hline 160 & 826 & 1262 & 1378 & 1702 & 1816 & 2256 \\
\hline 164 & 826 & 1262 & 1378 & 1702 & 1816 & 2256 \\
\hline 168 & 826 & 1262 & 1378 & 1702 & 1816 & 2256 \\
\hline 172 & 826 & 1262 & 1378 & 1702 & 1816 & 2256 \\
\hline 176 & 826 & 1262 & 1378 & 1702 & 1816 & 2256 \\
\hline 180 & 826 & 1262 & 1378 & 1702 & 1816 & 2256 \\
\hline 184 & 826 & 1262 & 1378 & 1702 & 1816 & 2256 \\
\hline 188 & 826 & 1262 & 1378 & 1702 & 1816 & 2256 \\
\hline 192 & 826 & 1262 & 1378 & 1702 & 1816 & 2256 \\
\hline 196 & 826 & 1262 & 1378 & 1702 & 1816 & 2256 \\
\hline
\end{tabular}


Tabela 3.8. (Cont.) Limites superior e inferior para os intervalos de confiança com coeficiente de confiança de $95 \%$ para pontos escolhidos nas curvas de referência aproximadas de $10 \%, 50 \%$ e $90 \%$.

\begin{tabular}{|c|c|c|c|c|c|c|}
\hline \multirow[b]{2}{*}{ Tempo } & \multicolumn{2}{|c|}{$\mathrm{Y}_{10 \%}$} & \multicolumn{2}{|c|}{$Y_{50 \%}$} & \multicolumn{2}{|c|}{$\mathrm{Y}_{90 \%}$} \\
\hline & L. inferior & L. superior & L.inferior & L. superior & L.inferior & L. superior \\
\hline 200 & 826 & 1262 & 1378 & 1702 & 1816 & 2256 \\
\hline 204 & 826 & 1262 & 1378 & 1702 & 1816 & 2256 \\
\hline 208 & 826 & 1262 & 1378 & 1702 & 1816 & 2256 \\
\hline 212 & 826 & 1262 & 1378 & 1702 & 1816 & 2256 \\
\hline 216 & 826 & 1262 & 1378 & 1702 & 1816 & 2256 \\
\hline 220 & 826 & 1262 & 1378 & 1702 & 1816 & 2256 \\
\hline 224 & 826 & 1262 & 1378 & 1702 & 1816 & 2256 \\
\hline 228 & 826 & 1262 & 1378 & 1702 & 1816 & 2256 \\
\hline 232 & 826 & 1262 & 1378 & 1702 & 1816 & 2256 \\
\hline 236 & 826 & 1262 & 1378 & 1702 & 1816 & 2256 \\
\hline 240 & 826 & 1262 & 1378 & 1702 & 1816 & 2256 \\
\hline 244 & 826 & 1262 & 1378 & 1702 & 1816 & 2256 \\
\hline 248 & 826 & 1262 & 1378 & 1702 & 1816 & 2256 \\
\hline 252 & 826 & 1262 & 1378 & 1702 & 1816 & 2256 \\
\hline 256 & 826 & 1262 & 1378 & 1702 & 1816 & 2256 \\
\hline 260 & 826 & 1262 & 1378 & 1702 & 1816 & 2256 \\
\hline 264 & 826 & 1262 & 1378 & 1702 & 1816 & 2256 \\
\hline 268 & 826 & 1262 & 1378 & 1702 & 1816 & 2256 \\
\hline 272 & 826 & 1262 & 1378 & 1702 & 1816 & 2256 \\
\hline 276 & 826 & 1262 & 1378 & 1702 & 1816 & 2256 \\
\hline 280 & 826 & 1262 & 1378 & 1702 & 1816 & 2256 \\
\hline 284 & 826 & 1262 & 1378 & 1702 & 1816 & 2256 \\
\hline 288 & 826 & 1262 & 1378 & 1702 & 1816 & 2256 \\
\hline 292 & 826 & 1262 & 1378 & 1702 & 1816 & 2256 \\
\hline 296 & 826 & 1262 & 1378 & 1702 & 1816 & 2256 \\
\hline 300 & 826 & 1262 & 1378 & 1702 & 1816 & 2256 \\
\hline 304 & 826 & 1262 & 1378 & 1702 & 1816 & 2256 \\
\hline 308 & 826 & 1262 & 1378 & 1702 & 1816 & 2256 \\
\hline 312 & 826 & 1262 & 1378 & 1702 & 1816 & 2256 \\
\hline 316 & 826 & 1262 & 1378 & 1702 & 1816 & 2256 \\
\hline 320 & 826 & 1262 & 1378 & 1702 & 1816 & 2256 \\
\hline 324 & 826 & 1262 & 1378 & 1702 & 1816 & 2256 \\
\hline 328 & 826 & 1262 & 1378 & 1702 & 1816 & 2256 \\
\hline 332 & 826 & 1262 & 1378 & 1702 & 1816 & 2256 \\
\hline 336 & 826 & 1262 & 1378 & 1702 & 1816 & 2256 \\
\hline 340 & 826 & 1262 & 1378 & 1702 & 1816 & 2256 \\
\hline 344 & 826 & 1262 & 1378 & 1702 & 1816 & 2256 \\
\hline 348 & 826 & 1262 & 1378 & 1702 & 1816 & 2256 \\
\hline 352 & 826 & 1262 & 1378 & 1702 & 1816 & 2256 \\
\hline 356 & 826 & 1262 & 1378 & 1702 & 1816 & 2256 \\
\hline 360 & 826 & 1262 & 1378 & 1702 & 1816 & 2256 \\
\hline
\end{tabular}


Figura 3.13. Curvas de referência aproximadas de $10 \%$, $50 \%$, e $90 \%$ e seus respectivos intervalos de confiança com coeficiente de confiança de $95 \%$.

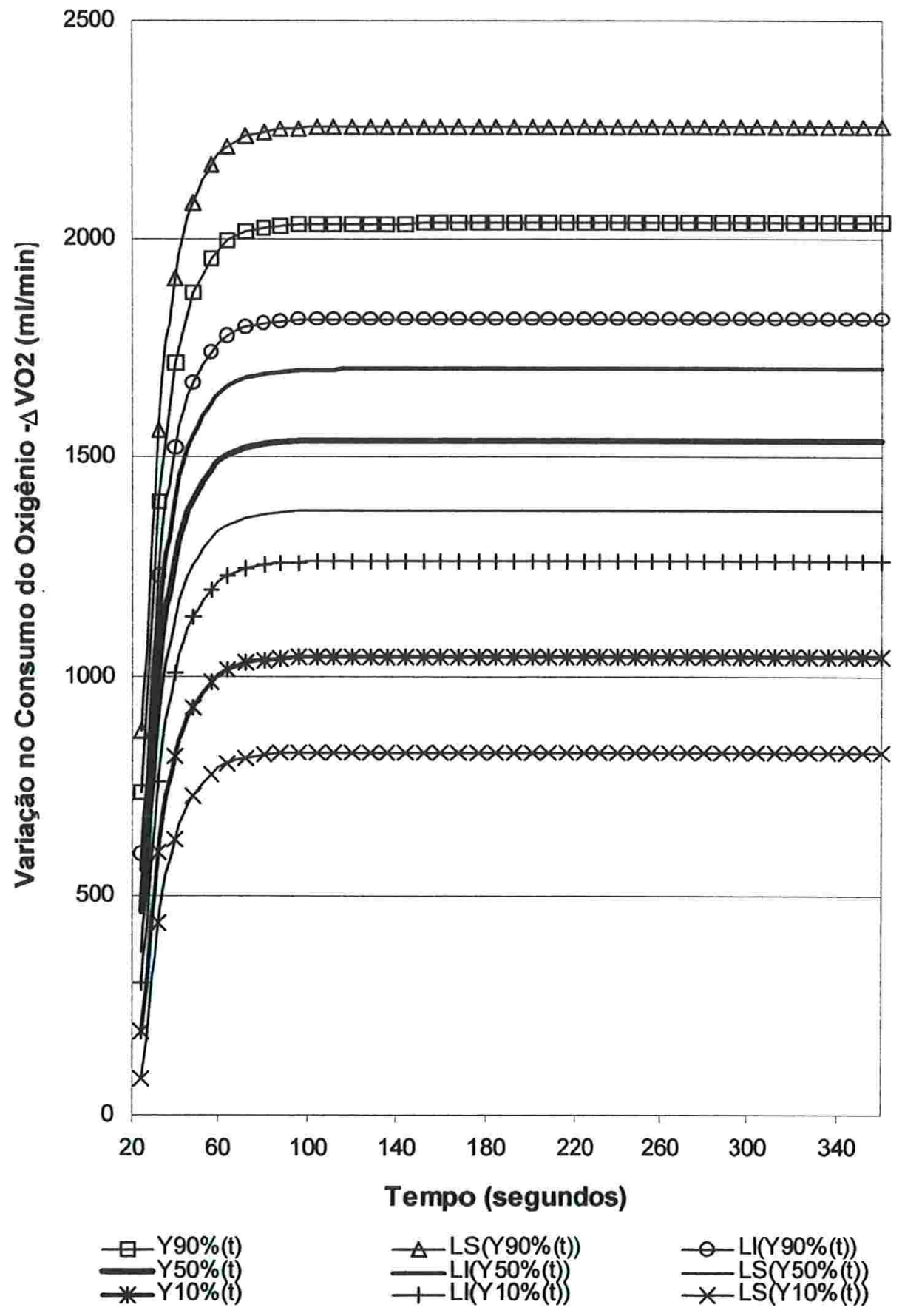




\section{Capítulo 4}

\section{Conclusão}

Neste trabalho uma abordagem para a construção de curvas de referência baseadas em modelos não linearizáveis para dados longitudinais foi considerada. Os procedimentos para a construção destas curvas de referência e de seus respectivos intervalos de confiança foram apresentados e exemplificados.

Esta proposta preenche uma lacuna relativa à construção de intervalos de referência e/ou curvas de referência existente na literatura. Considera-se uma abordagem inédita de construção de curvas de referência aproximadas para modelos não linearizáveis para dados longitudinais que corresponde a uma extensão dos resultados de Royston (1995) e Sañudo (2000).

O procedimento proposto baseia-se na metodologia de ajuste de modelos não lineares com efeitos aleatórios o que permite incorporar diferentes estruturas de covariância e dados não necessariamente balanceados e regularmente espaçados.

A construção das curvas de referência depende da especificação de um modelo não linear para descrever adequadamente os perfis de respostas. É desejável que este modelo não linear tenha uma justificativa teórica, embora também seja possível deduzi-lo por meio de uma análise descritiva. 
Supõe-se que o modelo probabilístico normal seja adequado para modelar a distribuição da característica de interesse em cada momento de observação.

Uma expressão para a verossimilhança da característica de interesse sob o modelo não linear com efeitos aleatórios é obtida através da especificação de um modelo aproximado, como em Beal and Sheiner (1985). Para tal, é necessário que o modelo seja diferenciável em relação aos efeitos aleatórios a fim de permitir uma expansão em série de Taylor de primeira ordem.

Finalmente, estimativas para os parâmetros do modelo são obtidas através da maximização da verossimilhança. Desse modo, as curvas de referência aproximadas e seus respectivos intervalos de referência são estimados por meio da substituição dos parâmetros envolvidos em suas equações pelas respectivas estimativas de máxima verossimilhança.

Outro ponto a destacar é a consideração da existência de dois usos distintos para as curvas de referência: identificação de valores extremos e perfis extremos. Especifica-se uma abordagem única para a construção destas curvas de referência, porém destaca-se que não se considera a variabilidade do termo de erro ao se calcular em curvas de referência destinadas à identificação de perfis extremos enquanto que para o cálculo das curvas de referência destinadas à identificação de valores extremos, considera-se toda a variabilidade dos dados.

Para trabalhos futuros, sugerem-se três linhas de pesquisa: (i) o desenvolvimento de técnicas para o diagnóstico do ajuste destas curvas de referência, (ii) o desenvolvimento de técnicas para a construção de curvas de referência baseadas em outros modelos probabilísticos e (iii) uma discussão e/ou o desenvolvimento de metodologia para a construção de intervalos de referência simultâneos para todos os pontos de uma dada curva de referência. 


\section{Referências Bibliográficas}

Altman, D. (1990). Practical Statistics for Medical Research. New York: Chapman and Hall/CRC.

Beal, S.L. and Sheiner, L.B. (1988). Heterocedastic Nonlinear Regression. Technometrics, 30, 327-338.

Davidian, M. and Giltinan, D.M. (1998). Nonlinear Models for Repeated Measurement Data. New York: Chapman and Hall/CRC.

Diggle, P.J.; Heagerty, P.; Liang, K. and Zeger, S.L. (2002). Analysis of Longitudinal Data, $2^{\text {nd }}$ ed. Oxford: Oxford University Press.

Fujita, M. (1998). Avaliação ultra-sonográfica do crescimento fetal em gestações gemelares. Dissertação de Doutorado. São Paulo: Faculdade de Medicina. Universidade de São Paulo, Brasil.

Fukuba, Y.; Hayashi, N.; Koga, S. and Yoshida, T.(2002). $\mathrm{VO}_{2}$ kinetics in heavy exercise is not altered by prior exercise with a different muscle group. Journal of Applied Physiology, 92, 2467-2474.

Gräsbeck, R. and Saris, N-E. (1969). Establishment and use of normal values. Scandinavian Journal of Clinical Laboratory Investigation, 24, 62-63.

Harris, E.K. and DeMets, D.L. (1972). Estimation of normal ranges and cumulative proportions by transforming observed distributions to Gaussian form. Clinical Chemistry, 18, 605-612.

Jones, R.H. (1993). Longitudinal Data with Serial Correlation: a Statespace Approach. London: Chapman and Hall.

Neter, J.; Kutner, M.H.; Nachtsheim, C.J. and Wasserman, W. (1996). Applied Linear Statistical Models, $4^{\text {th }}$. ed. Chicago: Irwin. 
Ocel Jr., J.V. (1997). Adaptation of the Slow Component of Vo2 Following 6 wk of High or Low Intensity Exercise Training. [cited in 2003, Aug $29^{\text {th }}$ ] Available from http://scholar.lib.vt.edu/theses/avaiable/etd10597-161936/unrestricted/jvodiss2.pdf.

Ogliari, P.J. and Andrade, D.F. (2001). Analysing longitudinal data via nonlinear models in randomized block designs. Computational Statistics \& Data Analysis, 36, 319-332.

Ratkowsky, D.A. (1989). Handbook of Nonlinear Regression Models. New York: Marcel Dekker, Inc.

Rossiter, H.B.; Howe, F.A.; Ward, S.A.; Kowalchuk, J.M.; Griffiths, J.R. and Whipp, B.J. (2000). Intersample fluctuations in phosphocreatine concentration determined by P-magnetic resonance spectroscopy and parameter estimation of metabolic response to exercise in humans. Journal of Physiology, 528, 359-369.

Rossiter, H.B.; Ward, S.A.; Kowalchuk, J.M.; Howe, F.A.; Griffiths, J.R. and Whipp, B.J.(2001). Effects of prior exercise on oxygen uptake and phosphocreatine kinetics during high-intensity knee-extension exercise in humans. Journal of Physiology, 537, 291-303.

Royston, P. and Matthews, J.N.S (1991). Estimation of reference ranges from normal samples. Statistics in Medicine, 10, 691-695.

Royston, P. (1991). Constructing time-specific reference ranges. Statistics in Medicine, 10, 675-690.

Royston, P. (1995). Calculation of unconditional and conditional reference intervals for foetal size and growth from longitudinal measurements. Statistics in Medicine, 14, 1417-1436.

Sañudo, A. (2000). Intervalos de confiança para curvas percentuais de peso fetal estimado em gestações gemelares. Dissertação de Mestrado. São Paulo: Instituto de Matemática e Estatística. Universidade de São Paulo, Brasil. 
SAS (2000). Version 8.02. Cary: SAS Institute Inc.

Sen, P.K. and Singer, J.M. (1993). Large Sample Methods in Statistics: An Introduction with Applications. New York: Chapman and Hall.

Singer, J.M. and Andrade, D.F. (2000). Analysis of Longitudinal Data. In Handbook of Statistics, C.R. Rao and P.K. Sen, (eds), 18, Amsterdam: North-Holland.

Singer, J.M. e Shinzato, A. R. (1999). Curva de peso fetal estimado em gestações gemelares. Relatório de Análise Estatística. São Paulo: Centro de Estatística Aplicada. Departamento de Estatística. Instituto de Matemática e Estatística. Universidade de São Paulo, Brasil.

Souza, G. S. (1998). Introdução aos Modelos de Regressão Linear e Nãolinear. Brasília: Embrapa.

Wright, E.M. and Royston, P. (1999). Calculating reference intervals for laboratory measurements. Statistical Methods in Medical Research, 8, 93-112. 